\title{
The Yang-Yang Thermodynamic Formalism and Large Deviations
}

\author{
T. C. Dorlas ${ }^{1}$ and J. T. Lewis, ${ }^{1}$ and J. V. Pulé ${ }^{2}$ \\ ${ }^{1}$ Dublin Institute for Advanced Studies, 10 Burlington Road, Dublin 4, Ireland \\ ${ }^{2}$ Dublin Institute for Advanced Studies, and Department of Mathematical Physics, University College, \\ Belfield, Dublin 4, Ireland
}

\begin{abstract}
The partition function for a one-dimensional system of Bosons with repulsive delta-function interaction is investigated. We prove that if the Bethe Ansatz eigenfunctions form a complete set then the grand canonical pressure is given by the Yang-Yang formula. The proof uses a probabilistic formalism to express the partition function as an expectation with respect to a probability measure on a Banach space of measures; the asymptotic behaviour of the expectation in the thermodynamic limit is determined by the Large Deviation Principle. This method is applicable in situations in which the Hamiltonian can be diagonalised using the Bethe Ansatz.
\end{abstract}

\section{Introduction}

Often, in mathematical physics, we are faced with the problem of determining the asymptotic behaviour, for large $l$, of a sequence

$$
\left\{\text { trace } \exp \left[-\beta \mathscr{H}^{l}\right] \mid l=1,2, \ldots\right\},
$$

where $\beta$ is a positive real number and $\left\{\mathscr{H}^{l} \mid l=1,2, \ldots\right\}$ is a sequence of self-adjoint operators on some Hilbert space. The problem arises, for example, in many-body theory; here $\mathscr{H}^{l}$ is the Hamiltonian of the system, $\beta$ is the inverse temperature and the volume $V_{l}$ of the system increases as $l$ increases. In this setting, there are not many cases in which the problem has been solved. For a long time, only for the free quantum gases, boson and fermion, was an explicit expression known for

$$
\lim _{l \rightarrow \infty} \frac{1}{\beta V_{l}} \ln \operatorname{trace} \exp \left[-\beta \mathscr{H}^{l}\right] .
$$

In 1969, Yang and Yang [1] made a notable advance: they developed a thermodynamic formalism for dealing with those interacting systems whose Hamiltonians can be diagonalized with the help of the Bethe Ansatz. Yang and Yang [1] applied their formalism to the quantum non-linear Schroedinger model whose Hamiltonian had been diagonalized six years previously by Lieb and Liniger [2]. In recent years, as more and more problems have succumbed to the Bethe 
Ansatz (see [3] or [4] for a review), the use of the Yang-Yang formalism has spread; for example, it has been used to determine the thermodynamic functions in the Kondo problem $[5,6,7]$ and to compute the central charge of the Virasoro algebra associated with critical two-dimensional classical statistical mechanical systems such as the Potts model and the Ashkin-Teller model [8].

The core of the Yang-Yang formalism is their derivation of an expression for the entropy density of the system; as they themselves point out, this derivation unlike the rest of their paper, is far from rigorous. It is, in fact, an ingenious elaboration of the derivation given by Landau and Lifshitz [9] for the nonequilibrium entropy density of a free quantum gas. Our aim in this paper is to give a rigorous proof of the Yang-Yang trace formula. We see little hope of doing this by supplying the needed rigour at each step of the Yang-Yang argument (any more than we could for the Landau-Lifshitz derivation). Instead, we use a probabilistic formalism to express trace $\exp \left[-\beta \mathscr{H}^{l}\right]$ as an integral

$$
\int_{E} e^{\beta V_{l} G[m]} \mathbf{K}_{l}[d m]
$$

with respect to a probability measure $\mathbf{K}_{l}$ on a topological space $E$ and we use Varadhan's theorem [10] to determine the asymptotic behaviour of the integral. Varadhan's theorem is an extension to regular topological spaces of Laplace's theorem on the asymptotic behaviour of integrals over the real line. By checking that the hypotheses of Varadhan's theorem are satisfied, we are able to give a rigorous proof of the Yang-Yang trace formula.

At first sight, the probabilistic formalism which we use may seem far removed from the Yang-Yang thermodynamic formalism. In fact, they are close in spirit, since Laplacian asymptotics (the method of the largest term) is at the heart of thermodynamics. Moreover, the Landau-Lifshitz expression for the non-equilibrium entropy density of a free Fermion gas appears naturally in the course of checking that the hypotheses of Varadhan's theorem are satisfied, and the Yang-Yang expression is related to it by a simple transformation.

In this paper, we apply the probabilistic formalism to the non-linear quantum Schroedinger model; we emphasize that it has the same wide applicability as has the Yang-Yang thermodynamic formalism. Nevertheless, it would not be profitable to display this work as an application of some general scheme, since the details may vary greatly from model to model. The classical non-linear Schroedinger model requires very different techniques; recently it has been treated rigorously by Lebowitz et al. [11].

Many-body theory is characterized by the existence of a number operator: for each Hamiltonian $\mathscr{H}^{l}$, there is a self-adjoint operator $\mathscr{N}^{l}$ whose spectrum is the set $0,1,2, \ldots$ and which commutes with $\mathscr{H}^{l}$. The operator $\mathscr{N}^{l}$ is interpreted as the observable corresponding to the total number of particles in the system; the eigenspace of $\mathscr{N}^{l}$ corresponding to the eigenvalue $N$ is called the $N$-particle subspace. Since $\mathscr{H}^{l}$ commutes with $\mathscr{N}^{l}$, we may regard $\mathscr{H}^{l}$ as the direct sum of a sequence $\left\{H_{N}^{l} \mid N=0,1,2, \ldots\right\}$ of operators, where $H_{N}^{l}$ is the restriction of $\mathscr{H}^{l}$ to the $N$-particle subspace. To investigate the asymptotic behaviour of trace $\exp \left\{-\beta \mathscr{H}^{l}\right\}$, it is convenient to generalize the problem slightly: we examine 
the behaviour of trace $\exp \left\{\beta\left(\mu \mathscr{N}^{l}-\mathscr{H}^{l}\right)\right\}$, where $\mu$ is a real number. Put

$$
p_{l}(\mu)=\frac{1}{\beta V_{l}} \ln \operatorname{trace} \exp \left\{\beta\left(\mu \mathcal{N}^{l}-\mathscr{H}^{l}\right)\right\},
$$

and, denoting by trace $_{N}$ the trace over the $N$-particle subspace, put

$$
f_{l}(\bar{\rho})=-\frac{1}{\beta V_{l}} \ln \operatorname{trace}_{N} \exp \left\{-\beta H_{N}^{l}\right\}
$$

where $\bar{\rho}=N / V_{l}$; we have

$$
\begin{aligned}
\exp \left\{\beta V_{l} p_{l}(\mu)\right\} & =\operatorname{trace} \exp \left\{\beta\left(\mu \mathcal{N}^{l}-\mathscr{H}^{l}\right)\right\} \\
& =\sum_{N=0,1,2, \ldots} e^{\beta \mu N} \operatorname{trace}_{N} \exp \left\{-\beta H_{N}^{l}\right\} \\
& =\sum_{N=0,1,2, \ldots} \exp \left\{\beta V_{l}\left(\mu \bar{\rho}-f_{l}(\bar{\rho})\right)\right\} .
\end{aligned}
$$

In many models of physical systems, the limits $p(\mu)=\lim _{l \rightarrow \infty} p_{l}(\mu)$ and $f(\bar{\rho})=$ $\lim _{l \rightarrow \infty} f_{l}(\bar{\rho})$ exist; the function $p(\cdot)$ is called the grand canonical pressure and the function $f(\cdot)$ is called the canonical free-energy. For $l$ sufficiently large, the main contribution to $p_{l}(\mu)$ comes from the largest term in the summation on the right-hand side of (1.3) and, in the limit, we have

$$
p(\mu)=\sup _{\bar{\rho}}\{\mu \bar{\rho}-f(\bar{\rho})\} .
$$

The crux of the thermodynamic formalism is the possibility of making an indirect evaluation of $f(\bar{\rho})$. We illustrate this first in the case of the free Fermion gas.

In the case of a free gas, the $N$-particle Hamiltonian $H_{N}^{l}$ is the sum of $N$ copies of the single-particle Hamiltonian $H_{1}^{l}$. Suppose that the single-particle Hamiltonian is the one-dimensional Laplacian with periodic boundary conditions on the interval $\left[0, V_{l}\right]$. The eigenvalues of $H_{N}^{l}$ are given by

$$
E_{N}^{l}(\mathbf{k})=|\mathbf{k}|^{2}=k_{1}^{2}+\cdots+k_{N}^{2}
$$

where

$$
k_{j}=\frac{2 \pi}{V_{l}} m_{j}, \quad m_{j} \in \mathbf{Z}
$$

In the case of Fermions, the $k_{j}$ are distinct: $k_{i} \neq k_{j}$ if $i \neq j$. As $l$ increases, $V_{l}$ increases and the possible values of the momenta $k_{j}$ become increasingly dense in the real line. It is argued that, in the limit $l \rightarrow \infty$ with $\bar{\rho}=N / V_{l}$ fixed, the "eigenvalues" are described, not by vectors $\mathbf{k}$, but by continuous distributions $\rho(\cdot)$. These are functions satisfying $\rho(k) \geqq 0, \int_{\mathbf{R}} \rho(k) d k / 2 \pi=\bar{\rho}$; the energy density corresponding to a distribution $\rho$ is given by

$$
u[\rho]=\int_{\mathbf{R}} k^{2} \rho(k) \frac{d k}{2 \pi}
$$

in the limit $l \rightarrow \infty$. But now we must count multiplicities. The entropy, the logarithm 
of the multiplicity of an eigenvalue, can be estimated in the limit $l \rightarrow \infty$ by a combinatorial argument (see Landau and Liftshitz [9], Sect. 54, p. 154) which gives its density as

$$
s[\rho]=-\int_{\mathbf{R}}\{\rho(k) \ln \rho(k)+(1-\rho(k)) \ln (1-\rho(k))\} \frac{d k}{2 \pi} .
$$

A second application of Laplacian asymptotics then gives the following expression for the free-energy density:

$$
f(\bar{\rho})=\inf _{\left\{\rho \in L_{+}^{1}(\mathbf{R})\|\rho\|_{1}=\bar{\rho}\right\}}\left\{u[\rho]-\beta^{-1} s[\rho]\right\} .
$$

This argument leads to the well-known formula

$$
p(\mu)=\beta^{-1} \int_{\mathbf{R}} \ln \left(1+e^{\beta\left(\mu-k^{2}\right)}\right) \frac{d k}{2 \pi}
$$

for the grand-canonical pressure of a free-Fermion gas.

Next we sketch briefly the extension of the thermodynamic formalism needed to deal with Hamiltonians which can be diagonalized with the aid of the Bethe Ansatz. Consider the non-linear quantum Schroedinger model: its Hamiltonian can be written symbolically as

$$
\mathscr{H}=\int_{\mathbf{R}}\left\{\partial_{x} \phi^{*}(x) \partial_{x} \phi(x)+2 c\left(\phi^{*}(x) \phi(x)\right)^{2}\right\} d x,
$$

where $\phi(x)$ is a one-dimensional Boson field satisfying

$$
\left[\phi(x), \phi^{*}(y)\right]=\delta(x-y),
$$

and $c \geqq 0$. The number operator $\mathscr{N}$ is given by

$$
\mathscr{N}=\int_{\mathbf{R}} \phi^{*}(x) \phi(x) d x
$$

It commutes with $\mathscr{H}$ and the restriction of $\mathscr{H}$ to the $N$-particle space, which we identify with $L^{2}\left(\mathbf{R}^{N}\right)_{\text {sym }}$, can be written as

$$
H_{N}=-\sum_{j=1}^{N} \partial_{x_{j}}^{2}+2 c \sum_{i>j} \delta\left(x_{i}-x_{j}\right)
$$

For $c=\infty$, we interpret $H_{N}$ to be $-\sum_{j=1}^{N} \partial_{x_{j}}^{2}$ with Dirichlet boundary conditions on the surfaces $x_{i}=x_{j},(i \neq j)$.

We restrict the system to a finite interval of length $V_{l}$, impose periodic boundary conditions and denote the resulting Hamiltonian by $H_{N}^{l}$. The eigenvalue problem for $H_{N}^{l}$ was solved by Lieb and Liniger [2], using the Bethe Ansatz. They obtain the remarkable result that the eigenvalues are given by

$$
\widetilde{E}_{N}^{l}(\mathbf{k})=\tilde{k}_{1}^{2}+\cdots+\tilde{k}_{N}^{2}
$$

with the $\tilde{k}_{j}$ solutions of the equations

$$
\tilde{k}_{j}=k_{j}-\frac{1}{V_{j}} \sum_{i=1}^{N} \theta_{c}\left(\tilde{k}_{j}-\tilde{k}_{i}\right)
$$


where

$$
\theta_{c}(k)=2 \arctan \left(\frac{k}{c}\right)
$$

and the $k_{j}$ are given by

and

$$
k_{j}=\frac{2 \pi}{V_{j}} m_{j}, \quad m_{j} \in \mathbf{Z}, \quad \text { if } N \text { is odd },
$$

$$
k_{j}=\frac{2 \pi}{V_{l}}\left(m_{j}+\frac{1}{2}\right), \quad m_{j} \in \mathbf{Z}, \quad \text { if } N \text { is even. }
$$

In other words, the eigenvalues of the $N$-particle Hamiltonian of the non-linear Schroedinger model can be labelled in the same way as the eigenvalues of the $N$-particle Hamiltonian of the free Fermion gas: there is a one-to-one correspondence between eigenvalues of $H_{N}^{l}$ and $N$-vectors $\mathbf{k}=\left(k_{1}, \ldots, k_{N}\right)$ with distinct entries taken, in this case, from the set $\left\{\ldots-2 \pi / V_{l}, 0,2 \pi / V_{l}, \ldots\right\}$ when $N$ is odd and from $\left\{\ldots,-3 \pi / V_{l},-\pi / V_{l}, \pi / V_{l}, 3 \pi / V_{l}, \ldots\right\}$ when $N$ is even. Yang and Yang [1] assumed that, just as in the free Fermion case, the "eigenvalues" can be described, in the limit $l \rightarrow \infty$ with $\bar{\rho}=N / V_{l}$ fixed, by a distribution $\rho(\tilde{k})$ satisfying

$$
\rho(\tilde{k}) \geqq 0, \quad \int_{\mathbf{R}} \rho(\tilde{k}) \frac{d \tilde{k}}{2 \pi}=\bar{\rho} .
$$

The energy density is now given by

$$
u[\rho]=\int_{\mathbf{R}} \tilde{k}^{2} \rho(\tilde{k}) \frac{d \tilde{k}}{2 \pi} .
$$

It remains to obtain an expression for the entropy density $s[\rho]$. In the free-Fermion case, we can interpret the term

$$
-\int_{\mathbf{R}} \rho(k) \ln \rho(k) \frac{d k}{2 \pi}
$$

as the contribution to the entropy density from the occupied $k$-values and the term

$$
-\int_{\mathbf{R}}(1-\rho(k)) \ln (1-\rho(k)) \frac{d k}{2 \pi}
$$

as the contribution from the unoccupied $k$-values (the "holes").

We could make this explicit by introducing $\rho_{h}$, the density of holes, and writing

$$
s[\rho]=\int_{\mathbf{R}}\left\{\left(\rho+\rho_{h}\right) \ln \left(\rho+\rho_{h}\right)-\rho \ln \rho-\rho_{h} \ln \rho_{h}\right\} \frac{d k}{2 \pi}
$$

together with the side-condition

$$
\rho(k)+\rho_{h}(k)=1 .
$$

In the case of the non-linear Schroedinger model, Yang and Yang give a combinatorial argument which, in the limit $l \rightarrow \infty$, yields the same formula (1.16) 
for the entropy density, but with the side-condition (1.17) replaced by

$$
\rho(\tilde{k})+\rho_{h}(\tilde{k})=1+\int_{\mathbf{R}} \theta_{c}^{\prime}(\tilde{k}-s) \rho(s) \frac{d s}{2 \pi} .
$$

Notice that, in the limit $c \rightarrow \infty$, the free-Fermion side-condition (1.17) is recovered. Using these expressions for $u[\rho]$ and $s[\rho]$, it is not difficult to solve the variational problem and obtain the Yang-Yang trace formula:

$$
\lim _{l \rightarrow \infty} \frac{1}{V_{l}} \ln \operatorname{trace} \exp \left\{-\beta \mathscr{H}^{l}\right\}=\int_{\mathbf{R}} \ln \left(1+e^{-\beta \varepsilon(k ; \beta)}\right) \frac{d k}{2 \pi},
$$

where $\varepsilon(k ; \beta)$ satisfies the integral equation

$$
\varepsilon(k ; \beta)=k^{2}-\beta^{-1} \int_{\mathbf{R}} \theta_{c}^{\prime}(k-s) \ln \left(1+e^{-\beta \varepsilon(s ; \beta)}\right) \frac{d s}{2 \pi} .
$$

Notice that, since $\theta_{c}^{\prime}(s)=2 c / c^{2}+s^{2}$, the free-Fermion result is recovered in the limit $c \rightarrow \infty$ and the free Boson result is recovered in the limit $c \rightarrow 0$.

We now turn to the probabilistic formalism. Our aim is to express

$$
\text { trace } \exp \left\{\beta\left(\mu \mathscr{N}^{l}-\mathscr{H}^{l}\right)\right\}
$$

in the case of the non-linear Schroedinger model, as an integral

$$
\int_{E} e^{\beta V_{l} G[x]} \mathbf{K}_{l}[d x]
$$

by suitable choices of topological space $E$, functional $G[\cdot]$ and probability measure $\mathbf{K}_{l}$. This will be accomplished using two propositions:

(1) In the case $c=\infty$, the limit

$$
p^{0}(\mu)=\lim _{l \rightarrow \infty} \frac{1}{\beta V_{l}} \ln \operatorname{trace} \exp \left\{\beta\left(\mu \mathcal{N}^{l}-\mathscr{H}^{l}\right)\right\}
$$

exists and is given by the free-Fermion expression

$$
p^{0}(\mu)=\beta^{-1} \int_{\mathbf{R}} \ln \left(1+e^{\beta\left(\mu-k^{2}\right)}\right) \frac{d k}{2 \pi} .
$$

(2) The eigenvalues of the Hamiltonian of the non-linear Schroedinger model for $0<c<\infty$ are in one-one correspondence with the eigenvalues of the Hamiltonian for $c=\infty$, and given by the Lieb-Liniger formula (1.14).

The first proposition is a well-known result; for completeness we give a proof in Sect. 2. Up to now, the status of the second proposition has been uncertain; the results presented in the Lieb-Liniger paper [2] are rigorous, but they do not claim that the Bethe Ansatz eigenfunction form a complete set; Yang and Yang [1] make such a claim, but only sketch an argument, based on continuity, to support it. Our proof of the Yang-Yang trace formula is complete modulo a proof of this proposition. We will return to the problem of completeness of the Bethe Ansatz eigenfunctions in another publication.

Since the strategy of proof which we adopt to verify the Yang-Yang thermo- 
dynamic formalism is not yet well-known among theoretical physicists, we first give an informal sketch of it. Consider, first, the case $c=\infty$ : We can write the trace (1.3) as a sum over configurations by introducing the space $\Omega$ defined by

$$
\Omega=\left\{\sigma: \mathbf{Z} \rightarrow\{0,1\} \mid \sum_{j \in \mathbf{Z}} \sigma_{j}<\infty\right\}
$$

and the functions $k^{l}: \Omega \rightarrow \mathbf{R}^{\mathrm{Z}}$ defined by

Then

$$
k^{l}(\sigma)_{n}= \begin{cases}\frac{2 \pi}{V_{l}} n, & \text { if } \sum_{j \in \mathbf{Z}} \sigma_{j} \text { is odd } \\ \frac{2 \pi}{V_{l}}\left(n+\frac{1}{2}\right), & \text { if } \sum_{j \in \mathbf{Z}} \sigma_{j} \text { is even }\end{cases}
$$

$$
\exp \left\{\beta V_{l} p_{l}^{0}(\mu)\right\}=\sum_{\sigma \in \Omega} \exp \left\{\beta \sum_{n \in \mathbf{Z}} \sigma_{n}\left(\mu-k^{l}(\sigma)_{n}^{2}\right)\right\} .
$$

Introducing the $c=\infty$-occupation measure on $\mathbf{R}$ by

$$
m_{l}[A ; \sigma]=\frac{1}{V_{l}} \sum_{n \in \mathbf{Z}} \sigma_{n} \delta_{k^{l}(\sigma)_{n}}[A], \quad A \subset \mathbf{R},
$$

we can re-write (1.25) as

$$
\exp \left\{\beta V_{l} p_{l}^{0}(\mu)\right\}=\sum_{\sigma \in \Omega} \exp \left\{\beta V_{l} \int_{\mathbf{R}}\left(\mu-k^{2}\right) m[d k ; \sigma]\right\} .
$$

The corresponding expression in the case $c<\infty$ is obtained by the following device: for an arbitrary bounded positive measure $m$, define the function $f_{m}$ as the unique solution of the equation

$$
f_{m}(k)=k-\int_{\mathbf{R}} \theta_{c}\left(f_{m}(k)-f_{m}\left(k^{\prime}\right)\right) m\left(d k^{\prime}\right)
$$

then we have

$$
\exp \left\{\beta V_{l} p_{l}(\mu)\right\}=\sum_{\sigma \in \Omega} \exp \left\{\beta V_{l} \int_{\mathbf{R}}\left(\mu-f_{m_{l}}(k)^{2}\right) m_{l}[d k ; \sigma]\right\} .
$$

(It is here that we have to assume that the Bethe Ansatz eigenstates form a complete set.) But this can be re-written as

$$
\begin{aligned}
\exp \left\{\beta V_{l} p_{l}(\mu)\right\} & =\sum_{\sigma \in \Omega} \exp \left\{\beta V_{l} \int_{\mathbf{R}}\left(k^{2}-f_{m_{l}}(k)^{2}\right) m_{l}[d k ; \sigma]\right\} \cdot \exp \left\{\beta V_{l} \int_{\mathbf{R}}\left(\mu-k^{2}\right) m_{l}[d k ; \sigma]\right\} \\
& =\exp \left\{\beta V_{l} p_{l}^{0}(\mu)\right\} \sum_{\sigma \in \Omega} \exp \left\{\beta V_{l} \int_{\mathbf{R}}\left(k^{2}-f_{m_{l}}(k)^{2}\right) m_{l}[d k ; \sigma]\right\} \mathbf{P}_{l}^{\mu}[\sigma],
\end{aligned}
$$

where $\mathbf{P}_{l}^{\mu}[$.$] is the probability measure defined on the countable set \Omega$ by

$$
\mathbf{P}_{l}^{\mu}[\sigma]=\exp \left\{-\beta V_{l} p_{l}^{0}(\mu)\right\} \exp \left\{\beta V_{l} \int_{\mathbf{R}}\left(\mu-k^{2}\right) m_{l}[d k ; \sigma]\right\} .
$$


This, in turn, induces a probability measure $\mathbf{K}_{l}^{\mu}$ on the space $E=$ $\left\{m \in \mathscr{M}_{b}^{+}(\mathbf{R}) \mid \int_{\mathbf{R}} k^{2} m(d k)<\infty\right\}$,

$$
\mathbf{K}_{l}^{\mu}=\mathbf{P}_{l}^{\mu} \circ m_{l}^{-1},
$$

since $m_{l}$ is a measurable mapping from $\Omega$ to $E$. Introducing the functional $G$ defined on $E$ by

$$
G[m]=\int_{\mathbf{R}}\left(k^{2}-f_{m}(k)^{2}\right) m(d k)
$$

we have, finally,

$$
\exp \left\{\beta V_{l} p_{l}(\mu)\right\}=\exp \left\{\beta V_{l} p_{l}^{0}(\mu)\right\} \int_{\mathbf{R}} e^{\beta V_{l} G[m]} \mathbf{K}_{l}^{\mu}[d m] .
$$

The measure $\mathbf{P}_{l}^{\mu}$ is in fact the grand canonical measure for $c=\infty$, and we call the induced measure $\mathbf{K}_{l}^{\mu}$ the Kac measure. (See introduction to [12] and [13] for the historical background.) But these interpretations carry no hidden hypotheses: for the purpose of the proof, $\mathbf{P}_{l}^{\mu}$ is the measure defined by (1.31).

The next step in the programme is to determine the asymptotic behaviour of $\mathbf{K}_{l}^{\mu}$ for large $l$. If we were able to define Lebesgue measure on $E$, we might aim to prove that, for large $l, \mathbf{K}_{l}^{\mu}$ behaves as $\exp \left\{-\beta V_{l} I^{\mu}[m]\right\} d m$ for some non-negative functional $I^{\mu}[m]$, and then apply Laplace's theorem to conclude that

$$
\lim _{l \rightarrow \infty} \frac{1}{\beta V_{l}} \ln \int_{E} e^{\beta V_{l} G[m]} \mathbf{K}_{l}^{\mu}[d m]=\sup _{E}\left\{G[m]-I^{\mu}[m]\right\} .
$$

In the absence of a suitable reference measure on $E$ we have to settle for a more technical description of the asymptotic behaviour of $\mathbf{K}_{l}^{\mu}$ :

Definition. Let $\left\{\mathbf{K}_{l} \mid l=1,2, \ldots\right\}$ be a sequence of Radon probability measures on a regular Hausdorff space $E$ and let $\left\{a_{l} \mid l=1,2, \ldots\right\}$ be an increasing sequence of positive numbers diverging to $+\infty$. The sequence $\left\{\mathbf{K}_{l}\right\}$ is said to obey the large deviation principle with constants $\left\{a_{l}\right\}$ and rate function $I: E \rightarrow[0, \infty]$ if the following conditions are satisfied:

(LD.1) $I[$.$] is lower semi-continuous.$

(LD.2) The level sets $\{x \in E \mid I[x] \leqq b\}$ with $0 \leqq b<\infty$ are compact.

(LD.3) For each closed set $C \subset E$,

$$
\limsup _{l \rightarrow \infty} \frac{1}{a_{l}} \ln K_{l}[C] \leqq-\inf _{x \in C}[x] .
$$

(LD.4) For each open set $O \subset E$,

$$
\liminf _{l \rightarrow \infty} \frac{1}{a_{l}} \ln \mathbf{K}_{l}[O] \geqq-\inf _{x \in O} I[x] .
$$

In place of the Laplace theorem, we have Varadhan's theorem. We state a version which covers all the situations that arise in this paper: 
Varadhan's Theorem. Let $\left\{\mathbf{K}_{l} \mid l=1,2, \ldots\right\}$ be a sequence of Radon probability measures on a regular Hausdorff space $E$ satisfying the large deviation principle with rate function $I: E \rightarrow[0, \infty]$, and constants $\left\{a_{l} \mid l=1,2, \ldots\right\}$. Suppose that $G: E \rightarrow \mathbf{R}$ is continuous and

$$
\lim _{A \rightarrow \infty} \limsup _{l \rightarrow \infty} \frac{1}{a_{l}} \ln \int_{\{x \in E \mid G(x) \geqq A\}} e^{a_{l} G(x)} \mathbf{K}_{l}[d x]=-\infty .
$$

Then

$$
\lim _{l \rightarrow \infty} \frac{1}{a_{l}} \ln \int_{E} e^{a_{l} G(x)} \mathbf{K}_{l}[d x]=\sup _{x \in E}\{G(x)-I(x)\} .
$$

We prove in Sect. 3 that the sequence $\left\{\mathbf{K}_{l}^{\mu}\right\}$ satisfies the large deviation principle with constants $\beta V_{l}$, and rate function $I^{\mu}[$.$] , where$

$$
I^{\mu}[m]=p^{0}(\mu)+f^{0}[m]-\mu\|m\| .
$$

Here $p^{0}(\mu)$ is the free Fermion pressure (1.22), and $f^{0}[\mathrm{~m}]$ is the free-Fermion free energy,

$$
f^{0}[m]=u[m]-\beta^{-1} s[m],
$$

where $u[m]=\int_{\mathbf{R}} k^{2} m(d k)$ is the internal energy and $s[m]$ is the entropy density,

$$
s[m]= \begin{cases}-\int_{\mathbf{R}}\{\rho \ln \rho+(1-\rho) \ln (1-\rho)\} \frac{d k}{2 \pi}, & \text { if } m(d k)=\rho(k) \frac{d k}{2 \pi} \text { and } \rho(k) \leqq 1 \\ -\infty & \text { otherwise. }\end{cases}
$$

After some reduction Varadhan's theorem yields the formula

$$
p(\mu)=\sup _{m \in E}\{\mu\|m\|-f[m]\},
$$

where

$$
f[m]=\int_{\mathbf{R}} f_{m}(k)^{2} m(d k)-\beta^{-1} s[m] .
$$

Using (1.40) it is not difficult to show that

$$
p(\mu)=\beta^{-1} \int_{\mathbf{R}} \ln \left(1+e^{-\beta \varepsilon(k ; \beta, \mu)}\right) \frac{d k}{2 \pi},
$$

where $\varepsilon(k ; \beta, \mu)$ satisfies the integral equation

$$
\varepsilon(k ; \beta, \mu)=k^{2}-\mu-\beta^{-1} \int_{\mathbf{R}} \theta_{c}^{\prime}(k-s) \ln \left(1+e^{-\beta \varepsilon(s ; \beta, \mu)}\right) \frac{d s}{2 \pi} .
$$

In this way the Yang-Yang trace formula is established. We recognize (1.39) as the Landau-Lifschitz expression for the free-Fermion entropy density.

There is an alternative expression for the local free energy $f[\mathrm{~m}]$ which makes the connection with the Yang-Yang result a little clearer: for an arbitrary measure 
$m$, define the function $h_{m}$ by

$$
h_{m}(k)=k+\int_{\mathbf{R}} \theta_{c}\left(k-k^{\prime}\right) m\left(d k^{\prime}\right) .
$$

In Sect. 2 we show that $h_{m}$ is the inverse of $f_{m}$. Defining

$$
\tilde{m}=m \circ f_{m}^{-1}
$$

we have

$$
u[m]=\int_{\mathbf{R}} f_{m}(k)^{2} m(d k)=\int_{\mathbf{R}} \tilde{k}^{2} \tilde{m}(d \tilde{k}) .
$$

When $\tilde{m}(d \tilde{k})=\tilde{\rho}(\tilde{k})(d \tilde{k} / 2 \pi)$, we have

$$
\rho(k)=\left(\tilde{\rho} \circ f_{m}\right)(k) f_{m}^{\prime}(k)=\tilde{\rho}(\tilde{k}) h^{\prime}(\tilde{k})^{-1}
$$

so that

$$
\begin{aligned}
s[m]= & -\int_{\mathbf{R}}\left\{\tilde{\rho}(\tilde{k})\left(\ln \tilde{\rho}(\tilde{k})-\ln h^{\prime}(\tilde{k})\right)\right. \\
& \left.+\left(1-\tilde{\rho}(\tilde{k}) h^{\prime}(\tilde{k})^{-1}\right) \ln \left(1-\tilde{\rho}(\tilde{k}) h^{\prime}(\tilde{k})^{-1}\right)\right\} h^{\prime}(\tilde{k}) \frac{d \tilde{k}}{2 \pi} \\
= & \int_{\mathbf{R}}\left\{h^{\prime}(\tilde{k}) \ln h^{\prime}(\tilde{k})-\tilde{\rho}(\tilde{k}) \ln \tilde{\rho}(\tilde{k})-\left(h^{\prime}(\tilde{k})-\tilde{\rho}(\tilde{k})\right) \ln \left(h^{\prime}(\tilde{k})-\tilde{\rho}(\tilde{k})\right)\right\} \frac{d \tilde{k}}{2 \pi} .
\end{aligned}
$$

Making the identification $\tilde{\rho}(\tilde{k})=\rho(\tilde{k})$ and $h^{\prime}(\tilde{k})-\tilde{\rho}(\tilde{k})=\rho_{h}(\tilde{k})$, we see that $(1.44)$ and (1.48) together are equivalent to the Yang-Yang expression, (1.16) and (1.18), for the entropy.

The advantage of the probabilistic formalism which we have sketched is that we are able to make each step rigorous. The first objective is to prove that the large deviation principle holds for the sequence $\left\{\mathbf{K}_{l}^{\mu}\right\}$ of Kac measures for free Fermions. To do this, we first find a candidate for the rate function. When $E$ is a topological vector space, there is a standard trick which often works: if $\left\{\mathbf{K}_{l}^{\mu}\right\}$ were to satisfy the large deviation principle with some rate function $I^{\mu}[$.$] and Varadhan's$ theorem were to hold for the linear functional $G[m]=\langle t, m\rangle$, then we would have

$$
\begin{aligned}
C^{\mu}[t] & =\lim _{l \rightarrow \infty} C_{l}^{\mu}[t]=\frac{1}{\beta V_{l}} \ln \int_{\boldsymbol{E}} e^{\beta V_{l}\langle t, m\rangle} \mathbf{K}_{l}^{\mu}[d m] \\
& =\sup _{m \in E}\left\{\langle t, m\rangle-I^{\mu}[m]\right\} .
\end{aligned}
$$

This relationship between $C^{\mu}[$.$] and I^{\mu}[$.$] is satisfied by the Legendre transform$ of $C^{\mu}$,

$$
I^{\mu}[m]=\sup _{t \in E_{*}}\left\{\langle t, m\rangle-C^{\mu}[t]\right\}
$$

so this expression is the usual starting point of a rigorous proof of the large deviation property. (See [13] for a counterexample.)

Here is the structure of the paper: 
In Sect. 2 we define carefully the sequence $\left\{\mathbf{K}_{l}^{\mu}\right\}$ of Kac measures for $c=\infty$ and calculate the limit $C^{\mu}$ of the sequence of cumulant generating functionals (Proposition 2.1). As we have seen, the Legendre transform (1.50) of $C^{\mu}$ is an obvious candidate for the rate function $I^{\mu}$ of the sequence $\left\{\mathbf{K}_{l}^{\mu}\right\}$, but first we must find a more useful expression for $I^{\mu}$; this is carried out in Sect. 3 where formula (1.37) is established (Theorem 3.1). In Sect. 4 we prove (Theorem 4.2) that the sequence $\left\{\mathbf{K}_{l}^{\mu}\right\}$ satisfies the large deviation principle with rate function $I^{\mu}$. The results of Sects. 2, 3, and 4 concern the $c=\infty-$ Kac measures and are of independent interest since trivial modifications yield the same results for the free-Fermion Kac measures. The remaining sections are concerned with checking that the other hypotheses of Varadhan's theorem hold. First we prove (Proposition 5.3) that the finite-volume trace, given by (1.29), is well-defined; then we prove (Proposition 6.2) that the functional $m \mapsto G[m]$, given by (1.33), is continuous; finally, in Sect. 7, we put it all together and prove the Yang-Yang trace formula. The main result of this paper is the following:

Theorem. Let $\mathscr{H}^{l}$ be the Hamiltonian of the quantum non-linear Schroedinger model on the interval $\left[0, V_{l}\right]$ and let $H_{N}^{l}$ be its restriction to the $N$-particle subspace. Then, assuming that the eigenvalues of $H_{N}^{l}$ are given by (1.13), we have, for all $\beta \in(0, \infty)$,

$$
\lim _{l \rightarrow \infty} \frac{1}{V_{l}} \ln \operatorname{trace} \exp \left\{-\beta \mathscr{H}^{l}\right\}=\int_{\mathbf{R}} \ln \left(1+e^{-\beta \varepsilon(k ; \beta)}\right) \frac{d k}{2 \pi},
$$

where $\varepsilon(\cdot ; \beta)$ is the unique solution of the equation

$$
\varepsilon(k ; \beta)=k^{2}-\beta^{-1} \int_{\mathbf{R}} \frac{2 c}{c^{2}+(k-s)^{2}} \ln \left(1+e^{-\beta \varepsilon(s ; \beta)}\right) \frac{d s}{2 \pi} .
$$

\section{Definition of the Kac Measure}

In the introduction we have defined the underlying probability space $\Omega$ of occupation numbers:

$$
\Omega=\left\{\sigma: \mathbf{Z} \rightarrow\{0,1\} \mid \sum_{n \in \mathbf{Z}} \sigma_{n}<\infty\right\} .
$$

We endow it with the product topology of $\{0,1\}^{\mathbf{z}}$ : a sequence $\left\{\sigma^{(m)}\right\}_{m=1}^{\infty}$ in $\Omega$ converges to $\sigma$ if and only if, for all $n$, there exists $m_{n}$ such that $\sigma_{n}^{(m)}=\sigma_{n}$ for all $m \geqq m_{n} . \Omega$ is a countable subspace of $\{0,1\}^{\mathbf{Z}}$. It is useful to define $\Omega_{\text {odd }}$ and $\Omega_{\text {even }}$ by

$$
\Omega_{\text {odd }}=\left\{\sigma \in \Omega \mid \sum_{n \in \mathbf{Z}} \sigma_{n}=1(\bmod 2)\right\},
$$

and

$$
\Omega_{\text {even }}=\left\{\sigma \in \Omega \mid \sum_{n \in \mathbf{Z}} \sigma_{n}=0(\bmod 2)\right\} .
$$

Since $\{0,1\}^{\mathrm{Z}}$ is Hausdorff space and $\Omega$ is countable, every subset of $\Omega$ is a Borel subset: $\mathscr{B}(\Omega)=\mathscr{P}(\Omega)$. 
We define a wavevector $k^{(l)}$ as in (1.24): it is a map $k^{(l)}: \Omega \rightarrow \mathbf{R}^{\mathbf{Z}}$ given by

$$
k_{n}^{(l)}(\sigma)=\left\{\begin{array}{lll}
2 \pi n / V_{l}, & \text { if } & \sigma \in \Omega_{\text {odd }} \\
2 \pi\left(n+\frac{1}{2}\right) / V_{l}, & \text { if } & \sigma \in \Omega_{\text {even }}
\end{array}\right.
$$

The occupation measure $m_{l}$ is the map $m_{l}: \Omega \rightarrow \mathscr{M}_{+}^{b}(\mathbf{R})$, the positive, bounded Radon measures on $\mathbf{R}$, given by (1.26):

$$
m_{l}[A, \sigma]=\frac{1}{V_{l}} \sum_{n \in \mathbf{Z}} \sigma_{n} \delta_{k_{n}^{(l)}(\sigma)}[A],
$$

where $\delta_{x}$ is the Dirac measure supported at $x: \delta_{x}[A]=\left\{\begin{array}{lll}1 & \text { if } & x \in A \\ 0 & \text { if } & x \notin A\end{array}\right.$

We define the $c=\infty$ grand canonical measure $\mathbf{P}_{l}^{\mu}$ on $\Omega$ by

$$
\mathbf{P}_{l}^{\mu}[A]=e^{-\beta V_{l} p_{l}^{0}(\mu)} \cdot \sum_{n \in A} \exp \left\{\beta \sum_{n \in \mathbf{Z}} \sigma_{n}\left(\mu-k_{n}^{(l)}(\sigma)^{2}\right)\right\},
$$

where the $c=\infty$ pressure $p_{l}^{0}(\mu)$ is defined by

$$
p_{l}^{0}(\mu)=\frac{1}{\beta V_{l}} \ln \left[\sum_{\sigma \in \Omega} \exp \left\{\beta \sum_{n \in \mathbf{Z}} \sigma_{n}\left(\mu-k_{n}^{(l)}(\sigma)^{2}\right)\right\}\right] \text {. }
$$

We have

$$
\begin{aligned}
& \prod_{n \geqq 0}\left(1+\exp \left\{\beta\left(\mu-\left(\frac{2 \pi}{V_{l}}\right)^{2}\left(n+\frac{1}{2}\right)^{2}\right)\right\}\right) \\
& \prod_{n>0}\left(1+\exp \left\{\beta\left(\mu-\left(\frac{2 \pi}{V_{l}}\right)^{2} n^{2}\right)\right\}\right) \leqq \exp \left\{\beta V_{l} p_{l}^{0}(\mu)\right\} \\
& \leqq \prod_{n \geqq 0}\left(1+\exp \left\{\beta\left(\mu-\left(\frac{2 \pi}{V_{l}}\right)^{2} n^{2}\right)\right\}\right) \\
& \prod_{n>0}\left(1+\exp \left\{\beta\left(\mu-\left(\frac{2 \pi}{V_{l}}\right)^{2}\left(n-\frac{1}{2}\right)^{2}\right)\right\}\right)
\end{aligned}
$$

from which it follows that

$$
p^{0}(\mu)=\lim _{l \rightarrow \infty} p_{l}^{0}(\mu)=\frac{1}{\beta} \int_{\mathbf{R}} \ln \left(1+e^{\beta\left(\mu-k^{2}\right)}\right) \frac{d k}{2 \pi} .
$$

As explained in the introduction we want to transfer the measures $\mathbf{P}_{l}^{\mu}$ to the space

$$
E=\left\{m \in \mathscr{M}_{+}^{b}(\mathbf{R}) \mid \int k^{2} m(d k)<\infty\right\},
$$

which we equip with the weak topology induced by the functions $f \in F$, where

$$
F=\left\{f: \mathbf{R} \rightarrow \mathbf{R} \mid f(k)=\left(1+k^{2}\right) \phi(k) \text { with } \phi \in \mathscr{C}_{0}(\mathbf{R})\right\} .
$$

$\left(\mathscr{C}_{0}(\mathbf{R})\right.$ denotes the space of real continuous functions vanishing at infinity.) This is the weak-* topology; that is $E=F^{*}$ as a Banach space. Given $t \in F$ we define the functional $p_{l}^{0}[t]$ by 


$$
p_{l}^{0}[t]=\frac{1}{\beta V_{l}} \ln \sum_{\sigma \in \Omega} \exp \left\{\beta \sum_{n \in \mathbf{Z}} \sigma_{n}\left(t\left(k_{n}^{(l)}(\sigma)\right)-k_{n}^{(l)}(\sigma)^{2}\right)\right\} .
$$

Lemma 1. Let $t \in F$. Then $p^{0}[t]=\lim _{l \rightarrow \infty} p_{l}^{0}[t]$ exists and is given $b y$

$$
p^{0}[t]=\frac{1}{\beta} \int_{\mathbf{R}} \ln \left(1+e^{\beta\left(t(k)-k^{2}\right)}\right) \frac{d k}{2 \pi} .
$$

Proof. The integral in (2.11) clearly converges since, for $|k|$ large, $\left(1+k^{2}\right)^{-1}|t(k)|<\frac{1}{2}$. This also shows that the tails of the sum in (2.10) are small. But, for $|k| \leqq \Lambda$,

$$
\frac{1}{\beta V_{l|n|}} \sum_{V_{l} \Lambda / 2 \pi} \ln \left(1+\exp \left[\beta\left(t\left(2 \pi n / V_{l}\right)-\left(2 \pi n / V_{l}\right)^{2}\right)\right]\right)
$$

converges as a Riemann sum to the integral

$$
\frac{1}{\beta V_{l}} \int_{-\Lambda}^{\Lambda} \ln \left(1+e^{\beta\left(t(k)-k^{2}\right)}\right) \frac{d k}{2 \pi} .
$$

With the above remark that every subset of $\Omega$ is a Borel subset it follows immediately that the mapping $m_{l}: \Omega \rightarrow E$ is Borel. The image measure $m_{l}\left(\mathbf{P}_{l}^{\mu}\right)=\mathbf{K}_{l}^{\mu}$ is therefore well-defined on $E$ by

$$
\mathbf{K}_{l}^{\mu}[B]=\mathbf{P}_{l}^{\mu}\left[m_{l}^{-1}[B]\right],
$$

for $B \in \mathscr{B}(E)$. We are going to prove that the sequence $\left\{\mathbf{K}_{l}^{\mu}\right\}$ satisfies the large deviation property, and towards this end we prove

Proposition 1. For $t \in F$ put

$$
C_{l}^{\mu}[t]=\frac{1}{\beta V_{l}} \ln \int_{E} e^{\beta V_{l}\langle t, m\rangle} \mathbf{K}_{l}^{\mu}[d m] .
$$

Then $C^{\mu}[t]=\lim _{l \rightarrow \infty} C_{l}^{\mu}[t]$ exists and is given by

$$
C^{\mu}[t]=p^{0}[\mu+t]-p^{0}(\mu)
$$

Proof. We have

$$
\begin{aligned}
C_{l}^{\mu}[t] & =\frac{1}{\beta V_{l}} \ln \int_{\Omega} \exp \left\{\beta \sum_{n \in \mathbf{Z}} \sigma_{n} t\left(k_{n}^{(l)}(\sigma)\right)\right\} \mathbf{P}_{l}^{\mu}[d \sigma] \\
& =p_{l}^{0}[\mu+t]-p_{l}^{0}(\mu) \rightarrow p^{0}[\mu+t]-p^{0}(\mu) .
\end{aligned}
$$

\section{Properties of the Rate Function}

As we argued in Sect. 1, we have the following candidate for the rate function:

$$
I^{\mu}[m]=\sup _{t \in F}\left\{\langle t, m\rangle-C^{\mu}[t]\right\},
$$

where $F$ is the space of functions $f(k)=\left(1+k^{2}\right) \phi(k)$ when $\phi \in \mathscr{C}_{0}(\mathbf{R})$. In this section 
we derive an explicit expression for $I^{\mu}[m]$ and find some useful properties along the way. First of all we have

Lemma 3.1. If $I^{\mu}[m]<\infty$ then $m$ is absolutely continuous with respect to Lebesgue measure.

Proof. Suppose that the singular part $m_{s}$ of $m$ is not identically zero. Choose $A>1$ and let $K$ be a compact set of Lebesgue measure zero such that $m_{s}(K) \neq 0$. Choose an open set $U \supset K$ satisfying

$$
\frac{1}{\beta} \int_{U} \ln \left(1+e^{\beta\left(\mu+A-k^{2}\right)}\right) \frac{d k}{2 \pi}<1 .
$$

(This is possible since $K$ has Lebesgue measure zero.) By Urysohn's lemma there exists a continuous function $t$ satisfying $0 \leqq t(k) \leqq A$ for all $k$, and $t(k)=\left\{\begin{array}{lll}A & \text { for } & k \in K \\ 0, & \text { for } & k \in U^{c}\end{array}\right.$ With this function $t$ we have

$$
C^{\mu}[t]=p[\mu+t]-p(\mu)<\frac{1}{\beta} \int_{U} \ln \left(1+e^{\beta\left(\mu+A-k^{2}\right)}\right) \frac{d k}{2 \pi}<1,
$$

so that $\langle t, m\rangle-C^{\mu}[t] \geqq A m_{s}(K)-1$. Taking $A \rightarrow \infty$ we find $I^{\mu}[m]=\infty$.

Note that, when $t$ is continuous with compact support $K$ we have the estimate

$$
C^{\mu}[t]=\frac{1}{\beta} \int_{K} \ln \left(1+e^{\beta\left(\mu+t(k)-k^{2}\right)}\right) \frac{d k}{2 \pi} \leqq \frac{1}{2 \pi}\left(\mu+\|t\|_{\infty}\right)|K| .
$$

Lemma 3.2. If $I^{\mu}[m]<\infty$ and $m$ has a density $(2 \pi)^{-1} \rho$ with respect to Lebesgue measure, then $0 \leqq \rho(k) \leqq 1$ for almost all $k$.

Proof. Let $m(d k)=\rho(k)(d k / 2 \pi)$ and suppose that there exists a subset $S \subset \mathbf{R}$ with positive Lebesgue measure: $|S|>0$, such that $\rho(k) \geqq \rho_{0}>1$ for all $k \in S$. Given $\varepsilon>0$ we choose $C \subset S$ compact and $O \supset S$ open such that $|O \backslash C|<\varepsilon$ and we take $t \in \mathscr{C}(\mathbf{R})$ such that $0 \leqq t(k) \leqq A$ and $t(k)=\left\{\begin{array}{lll}A, & \text { for } & k \in C ; \\ 0 & \text { for } & k \in O^{c}\end{array}\right.$ Then

$$
\begin{aligned}
\langle t, m\rangle-C^{\mu}[t] & \geqq \frac{1}{2 \pi} \rho_{0} A|C|-\frac{1}{2 \pi}(|\mu|+A)|O| \\
& \geqq \frac{1}{2 \pi} A\left\{\left(\rho_{0}-1\right)|S|-\left(\rho_{0}+1\right) \varepsilon\right\}-\frac{1}{2 \pi} \mu(|S|+\varepsilon),
\end{aligned}
$$

using (3.2). Since $\rho_{0}>1$ we can choose $\varepsilon$ so that $\left(\rho_{0}-1\right)|S|-\left(\rho_{0}+1\right) \varepsilon>0$. Letting $A \rightarrow \infty$ we conclude that $I^{\mu}[m]=\infty$ if $\rho(k)>1$ on a set of positive Lebesgue measure.

Lemma 3.3. Let $m \in E$ be such that $I^{\mu}[m]<\infty$. Then

$$
I^{\mu}[m]=\sup _{t \in \mathscr{L}^{1}}\left\{\langle t, m\rangle-C^{\mu}[t]\right\} .
$$


Proof. We first truncate a given $t \in F$ with

$$
\alpha_{n}(k)= \begin{cases}1, & \text { if } \quad|k| \leqq n \\ -|k|+n+1, & \text { if } n \leqq|k| \leqq n+1 \\ 0, & \text { if }|k| \geqq n+1\end{cases}
$$

and put $t_{n}=\alpha_{n} t$. Then $t_{n} \in \mathscr{C}_{c}(\mathbf{R})$ and $\left\langle t_{n}, m\right\rangle \rightarrow\langle t, m\rangle$ and also $C^{\mu}\left[t_{n}\right] \rightarrow C^{\mu}[t]$. Therefore $I^{\mu}\left(m, t_{n}\right) \rightarrow I^{\mu}(m, t)$. We conclude that

$$
I^{\mu}[m]=\sup _{t \in \mathscr{C}_{c}(\mathbf{R})}\left\{\langle t, m\rangle-C^{\mu}[t]\right\} .
$$

Now $\mathscr{C}_{c}(\mathbf{R})$ is dense in $\mathscr{L}^{1}$. Let $t \in \mathscr{L}^{1}$. Then $\langle t, m\rangle$ is well-defined since $m(d k)=$ $\rho(k)(d k / 2 \pi)$ with $\rho \in \mathscr{L}^{\infty}$, and $C^{\mu}[t]=p^{0}[\mu+t]-p^{0}(\mu)$ is well-defined because, if $t_{n} \in \mathscr{C}_{c}(\mathbf{R})$ and $t_{n} \rightarrow t$ in $\mathscr{L}^{1}$, then $\left|C^{\mu}\left[t_{n}\right]-C^{\mu}[t]\right| \leqq \int\left|t_{n}(k)-t(k)\right|(d k / 2 \pi)$ using the fact that $\left|\ln \left(1+e^{x}\right)-\ln \left(1+e^{y}\right)\right| \leqq|x-y|$. We also have $\left|\left\langle t_{n}, m\right\rangle-\langle t, m\rangle\right| \leqq$ $(1 / 2 \pi)\left\|t_{n}-t\right\|_{1}$ because $0 \leqq \rho(k) \leqq 1$. This shows that $I^{\mu}\left(m, t_{n}\right) \rightarrow I^{\mu}(m, t)$, which proves the lemma.

Lemma 3.4. Define, for $t \in F$ or $t \in \mathscr{L}^{1}$,

$$
\rho^{\mu+t}(k)=\frac{1}{1+\exp \left\{\beta\left(k^{2}-\mu-t(k)\right)\right\}} .
$$

The Fermi-Dirac measures $m^{\mu+t}(d k)=\rho^{\mu+t}(k)(d k / 2 \pi)$ satisfy

$$
I^{\mu}\left[m^{\mu+t}\right]=\left\langle t, m^{\mu+t}\right\rangle-C^{\mu}[t] .
$$

Proof. Clearly $I^{\mu}\left[m^{\mu+t}\right] \geqq\left\langle t, m^{\mu+t}\right\rangle-C^{\mu}[t]$. It remains to show that, for any other $\tilde{t} \in F$ respectively $\tilde{t} \in \mathscr{L}^{1},\left\langle t, m^{\mu+t}\right\rangle-C^{\mu}[t] \geqq\left\langle\tilde{t}, m^{\mu+t}\right\rangle-C^{\mu}[\tilde{t}]$. Given $\alpha, x \in \mathbf{R}$ define $f_{\alpha}(x, k)=x \rho^{\mu+\alpha}(k)-(1 / \beta) \ln \left(1+e^{\beta\left(\mu+x-k^{2}\right)}\right)$. Then $x \mapsto f_{\alpha}(x, k)$ is concave and $x=\alpha$ is a stationary point. Hence $f_{\alpha}(x, k) \leqq f_{\alpha}(\alpha, k)$. Now put $\alpha=t(k)$ and $x=\tilde{t}(k)$, and integrate.

Let us define an entropy function $s(x)$ by

$$
s(x)= \begin{cases}-x \ln x-(1-x) \ln (1-x), & \text { if } 0<x<1 \\ 0, & \text { if } x=0 \text { or } x=1 .\end{cases}
$$

We then have the following identity:

$$
s\left(\rho^{\mu}(k)\right)=\beta \rho^{\mu}(k)\left(k^{2}-\mu\right)+\ln \left(1+e^{\beta\left(\mu-k^{2}\right)}\right) .
$$

Inserting in (3.4) we obtain

$$
I^{\mu}\left[m^{\mu+t}\right]=p^{0}(\mu)+\int\left(k^{2}-\mu\right) m^{\mu+t}(d k)-\frac{1}{\beta} \int s\left(\rho^{\mu+t}(k)\right) \frac{d k}{2 \pi} .
$$

Next we show, by approximating a general $m$ by Fermi-Dirac measures, that this formula is generally valid.

Theorem 3.1. Let $m \in E$ be such that $I^{\mu}[m]<\infty$. Then

$$
I^{\mu}[m]=p^{0}(\mu)+f^{0}[m]-\mu\|m\|,
$$


where

$$
f^{0}[m]=\int_{\mathbf{R}} k^{2} m(d k)-\beta^{-1} s[m]
$$

and

$$
s[m]=-\int_{\mathbf{R}}\{\rho(k) \ln \rho(k)+(1-\rho(k)) \ln (1-\rho(k))\} \frac{d k}{2 \pi} .
$$

Proof. We first show that $I^{\mu}[m] \leqq f^{0}[m]+p^{0}(\mu)-\mu\|m\|$.

$$
\begin{aligned}
I^{\mu}[m] & =\sup _{t \in F}\left\{\int t(k) \rho(k) \frac{d k}{2 \pi}-\frac{1}{\beta} \int \ln \left(1+e^{\beta\left(\mu+t(k)-k^{2}\right)}\right) \frac{d k}{2 \pi}\right\}+p^{0}(\mu) \\
& \leqq \int \frac{d k}{2 \pi} \sup _{r \in \mathbf{R}}\left\{\tau \rho(k)-\frac{1}{\beta} \ln \left(1+e^{\beta\left(\mu+\tau-k^{2}\right)}\right)\right\}+p^{0}(\mu) \\
& =\int\left\{k^{2} \rho(k)-\frac{1}{\beta} s(\rho(k))\right\} \frac{d k}{2 \pi}+p^{0}(\mu)-\mu\|m\|=p^{0}(\mu)+f^{0}[m]-\mu\|m\|<\infty .
\end{aligned}
$$

To prove the reverse inequality we define $t_{n}^{M}$ as follows. Given $M>0$ and $n>1$ we subdivide the real line into the four regions: $R_{0}=\{k|| k \mid>M\}, R_{1}=\{k|| k \mid \leqq M$ and $\left.\rho(k)<\rho^{\mu-n}(k)\right\}, \quad R_{2}=\left\{k|| k \mid \leqq M \quad\right.$ and $\left.\quad \rho^{\mu-n}(k) \leqq \rho(k) \leqq 1-1 / n\right\}, \quad$ and $R_{3}=\{k|| k \mid \leqq M$ and $\rho(k)>1-1 / n\}$, where $\rho^{\mu}$ was defined in (3.3). We put

$$
t_{n}^{M}(k)=\left\{\begin{array}{lll}
0, & \text { if } & k \in R_{0} ; \\
k^{2}-\mu-\frac{1}{\beta} \ln \frac{1-\rho(k)}{\rho(k)}, & \text { if } & k \in R_{2} ; \\
-n, & \text { if } & k \in R_{1} \\
k^{2}-\mu+\ln (n-1), & \text { if } & k \in R_{3} .
\end{array}\right.
$$

Clearly $t_{n}^{M} \in \mathscr{L}^{1}$, so that by Lemma $3.3, I^{\mu}[m] \geqq\left\langle t_{n}^{M}, m\right\rangle-C^{\mu}\left[t_{n}^{M}\right]$. But

$$
\begin{aligned}
p^{0}(\mu) & +f^{0}[m]-\mu\|m\|-\left\langle t_{n}^{M}, m\right\rangle+C^{\mu}\left[t_{n}^{M}\right] \\
\leqq & \int_{R_{0}} \frac{d k}{2 \pi}\left\{\frac{1}{\beta} \ln \left(1+e^{\beta\left(\mu-k^{2}\right)}\right)+k^{2} \rho(k)-\frac{1}{\beta} s(\rho(k))-\mu \rho(k)\right\} \\
& +n \int_{R_{1}} \rho(k) \frac{d k}{2 \pi}+\frac{1}{\beta} \int_{R_{1}} \ln \left(1+e^{\beta\left(\mu-n-k^{2}\right)}\right) \frac{d k}{2 \pi} \\
& +\int_{R_{1}} k^{2} \rho(k) \frac{d k}{2 \pi}-\frac{1}{\beta} \int_{R_{1}} s(\rho(k)) \frac{d k}{2 \pi}-\mu \int_{R_{1}} \rho(k) \frac{d k}{2 \pi} \\
& -\frac{1}{\beta} \ln (n-1) \int_{R_{3}} \rho(k) \frac{d k}{2 \pi}+\frac{1}{\beta} \ln n \int_{R_{3}} \frac{d k}{2 \pi}-\frac{1}{\beta} \int_{R_{3}} s(\rho(k)) \frac{d k}{2 \pi} .
\end{aligned}
$$

The integral over $R_{0}$ approaches zero as $M \rightarrow \infty$ because the corresponding integral over $\mathbf{R}$ converges. Furthermore, we can omit all negative terms in the bound on the right-hand side of (3.12), and 


$$
\begin{aligned}
& n \int_{R_{1}} \rho(k) \frac{d k}{2 \pi} \leqq n \int \rho^{\mu-n}(k) \frac{d k}{2 \pi}=n \int \frac{1}{1+\exp \left\{\beta\left(k^{2}+n-\mu\right)\right\}} \frac{d k}{2 \pi} \rightarrow 0 \text { as } n \rightarrow \infty, \\
& \frac{1}{\beta} \int_{R_{2}} \ln \left(1+e^{\beta\left(\mu-n-k^{2}\right)}\right) \frac{d k}{2 \pi} \leqq p^{0}(\mu-n) \rightarrow 0 \text { as } n \rightarrow \infty,
\end{aligned}
$$

and

$$
\int_{R_{1}} k^{2} \rho(k) \frac{d k}{2 \pi} \leqq \int \frac{d k}{2 \pi} \frac{k^{2}}{1+\exp \left\{\beta\left(k^{2}+n-\mu\right)\right\}} \rightarrow 0 .
$$

We are left with

$$
\begin{aligned}
& \frac{1}{\beta}\left\{\ln n \int_{R_{3}} \frac{d k}{2 \pi}-\ln (n-1) \int_{R_{3}} \rho(k) \frac{d k}{2 \pi}\right\} \\
& \quad \leqq \frac{1}{\beta}\left\{\frac{1}{n-1} \ln n-\ln \left(1-\frac{1}{n}\right)\right\} \int_{R_{3}} \rho(k) \frac{d k}{2 \pi} \rightarrow 0 \quad(n \rightarrow \infty) .
\end{aligned}
$$

As a corollary we have

Theorem 3.2. (Approximation theorem). Let $m \in E$ be such that $I^{\mu}[m]<\infty$. Then there exists a sequence $t_{n} \in F, n=1,2, \ldots$ such that the corresponding Fermi-Dirac measures $m_{n}$ defined by

$$
m_{n}(d k)=\rho^{\mu+t_{n}}(k) \frac{d k}{2 \pi}
$$

satisfy

$$
\begin{aligned}
& \text { (a) } \lim _{n \rightarrow \infty} m_{n}=m \text { in } E, \\
& \text { (b) } \lim _{n \rightarrow \infty} I^{\mu}\left[m_{n}\right]=I^{\mu}[m] .
\end{aligned}
$$

Proof. Put $t_{n}=t_{n}^{M_{n}}$ as in (3.11), where $M_{n}$ is still to be determined. Then, by Lemma $3.4,\left\langle t_{n}, m_{n}\right\rangle-C^{\mu}\left[t_{n}\right]=I^{\mu}\left[m_{n}\right]$. We have shown that, as $n \rightarrow \infty$, $\left\langle t_{n}, m\right\rangle-C^{\mu}\left[t_{n}\right] \rightarrow I^{\mu}[m]$. But

$$
\begin{aligned}
\left|\left\langle t_{n}, m-m_{n}\right\rangle\right| \leqq & n \int_{R_{1}}\left|\rho(k)-\rho^{\mu+t_{n}}(k)\right| \frac{d k}{2 \pi} \\
& +\int_{R_{3}}\left|\rho(k)-\rho^{\mu+t_{n}}(k)\right|\left\{k^{2}+|\mu|+\frac{1}{\beta} \ln (n-1)\right\} \frac{d k}{2 \pi} .
\end{aligned}
$$

If $k \in R_{1}$ then $t_{n}(k)=-n$, so $\rho^{\mu+t_{n}}(k)=\rho^{\mu-n}(k)$. The first term on the right-hand side of (3.14) is therefore bounded by

$$
n \int\left(1+e^{\beta\left(k^{2}-\mu+n\right)}\right)^{-1} \frac{d k}{2 \pi} \rightarrow 0 .
$$

In the second term $\rho^{\mu+t_{n}}(k)=1-1 / n$, so that this term is bounded by 


$$
\begin{aligned}
& \frac{1}{n} \int_{R_{3}}\left\{k^{2}+|\mu|+\frac{1}{\beta} \ln (n-1)\right\} \frac{d k}{2 \pi} \\
& \quad \leqq \frac{1}{n-1} \int_{\rho(k) \geqq 1-1 / n} \rho(k)\left\{k^{2}+|\mu|+\frac{1}{\beta} \ln (n-1)\right\} \frac{d k}{2 \pi} \rightarrow 0 \text { as } n \rightarrow \infty .
\end{aligned}
$$

This proves (b) for the measures $m_{n}$ of the form (3.13) with $t_{n}=t_{n}^{M_{n}}$ of the form (3.11).

To prove (a) let $t \in F$ and write $\varphi(k)=\left(1+k^{2}\right)^{-1} t(k)$. Then

$$
\left|\left\langle t, m-m_{n}\right\rangle\right| \leqq \int_{R_{0} \cup R_{1} \cup R_{3}}|t(k)|\left|\rho(k)-\rho^{\mu+t_{n}}(k)\right| \frac{d k}{2 \pi} .
$$

The integral over $R_{0}$ vanishes as $M \rightarrow \infty$ because

$$
\int\left(1+k^{2}\right) \rho(k) \frac{d k}{2 \pi}<\infty
$$

and

$$
\int\left(1+k^{2}\right) \rho^{\mu}(k) \frac{d k}{2 \pi}=\int \frac{1+k^{2}}{1+\exp \left\{\beta\left(k^{2}+n-\mu\right)\right\}} \frac{d k}{2 \pi}<\infty .
$$

The second term is bounded by

$$
\|\varphi\|_{\infty} \int \frac{1+k^{2}}{1+\exp \left\{\beta\left(k^{2}+n-\mu\right)\right\}} \frac{d k}{2 \pi},
$$

which converges to zero as $n \rightarrow \infty$. The third term is bounded by

$$
\int_{R_{3}} k^{2}\left\{\rho(k)-\left(1-\frac{1}{n}\right)\right\} \frac{d k}{2 \pi} \leqq \frac{1}{n} \int_{|k| \leqq M_{n}} k^{2} \frac{d k}{2 \pi},
$$

which converges to zero as $n \rightarrow \infty$ when we choose $M_{n}=\sqrt[4]{n}$.

Next we approximate $t_{n}^{M}$ by $t \in \mathscr{C}_{c}(\mathbf{R}) \subset F$. Since $\mathscr{C}_{c}(\mathbf{R})$ is dense in $\mathscr{L}^{1}$, there exists, given $\varepsilon>0$, a $t \in \mathscr{C}_{c}(\mathbf{R})$ such that $\left\|t-t_{n}^{M}\right\|_{1}<\varepsilon$. In the proof of Lemma 3.3 we have seen that

$$
\left|C^{\mu}[t]-C^{\mu}\left[t_{n}^{M}\right]\right|<\frac{1}{2 \pi}\left\|t-t_{n}^{M}\right\|_{1}<\varepsilon / 2 \pi
$$

Also

$$
\begin{aligned}
\left|\left\langle t, m^{\mu+t}\right\rangle-\left\langle t_{n}^{M}, m^{\mu+t_{n}^{M}}\right\rangle\right| & \leqq\left|\left\langle t-t_{n}^{M}, m^{\mu+t}\right\rangle\right|+\left|\left\langle t_{n}^{M}, m^{\mu+t}\right\rangle-\left\langle t_{n}^{M}, m^{\mu+t_{n}^{M}}\right\rangle\right| \\
& \leqq \frac{1}{2 \pi}\left(1+\beta \sup _{k \in \mathbf{R}}\left|t_{n}^{M}(k)\right|\right) \varepsilon
\end{aligned}
$$

because

$$
\left|\rho^{\mu+t}(k)-\rho^{\mu+t_{n}^{M}}(k)\right| \leqq \beta\left|t(k)-t_{n}^{M}(k)\right| .
$$

Since $t_{n}^{M}$ is bounded we find, using Lemma 3.4, that

$$
\left|I^{\mu}\left[m^{\mu+t}\right]-I^{\mu}\left[m^{\mu+t_{n}^{M}}\right]\right| \leqq \text { const. } \varepsilon .
$$

This proves the approximation theorem. 


\section{The Large Deviation Property}

In the proof of the large deviation property for the Kac measure defined by (2.12) we need the large deviation property for the distribution of the mean density $\bar{\rho}$ and the mean energy $\varepsilon=\int k^{2} \rho(k) d k / 2 \pi$. This is a so-called first level large deviation result. The final result about the measure (2.12) is a second level large deviation property. Let us now state the first-level result. Let $\widetilde{\mathbf{K}}_{l}^{\mu}$ be the image measure of $\mathbf{K}_{l}^{\mu}$ for the mapping $m \rightarrow(\bar{\rho}, \varepsilon)$, where

$$
\bar{\rho}=\|m\|, \quad \text { and } \quad \varepsilon=\int k^{2} m(d k) .
$$

(Note that this mapping from $E$ to $[0, \infty) \times[0, \infty)$ is Borel but not continuous.)

Theorem 4.1. The sequence $\left\{\tilde{\mathbf{K}}_{l}^{\mu} \mid l=1,2, \ldots\right\}$ of probability measures on $[0, \infty) \times$ $[0, \infty)$ satisfies the large deviation property with constants $\beta V_{l}$ and rate function

$$
\tilde{I}^{\mu}(\bar{\rho}, \varepsilon)=\sup _{\substack{-\infty<\alpha<\infty \\-\infty<x<1}}\left\{\alpha \bar{\rho}+x \varepsilon-\tilde{C}^{\mu}(\alpha, x)\right\},
$$

where

$$
\tilde{C}^{\mu}(\alpha, x)=(1-x)^{-1 / 2} p^{0}(\mu+\alpha)-p^{0}(\mu) .
$$

Proof. The lower semicontinuity of $\widetilde{I}^{\mu}$ follows immediately from the expression (4.2) and the fact that $\widetilde{C}^{\mu}$ is continuous. Furthermore, $\widetilde{C}^{\mu}$ is convex, so that we can obtain the supremum in (4.2) by putting the partial derivatives equal to zero:

$$
\left\{\begin{array}{l}
\bar{\rho}=(1-x)^{-1 / 2} p^{0 \prime}(\mu+\alpha) \\
\varepsilon=\frac{1}{2}(1-x)^{-3 / 2} p^{0}(\mu+\alpha) .
\end{array}\right.
$$

Inserting into (4.2) we obtain, writing $\sigma=\alpha+\mu$,

$$
\tilde{I}^{\mu}(\bar{\rho}, \varepsilon)=\alpha(1-x)^{-1 / 2} p^{0 \prime}(\sigma)+\frac{1}{2} x(1-x)^{-3 / 2} p^{0}(\sigma)-(1-x)^{-1 / 2} p^{0}(\sigma)+p^{0}(\mu) .
$$

Clearly, if $\bar{\rho} \rightarrow \infty$ or $\varepsilon \rightarrow \infty$, then either $x \rightarrow 1$ or $\alpha \rightarrow \infty$. If $x \rightarrow 1$ then obviously $\widetilde{I}^{\mu}(\bar{\rho}, \varepsilon) \rightarrow \infty$. For $\alpha \rightarrow \infty$, we use the asymptotic behaviour of $p^{0}(\sigma)$ and $p^{0 \prime}(\sigma)$ :

$$
p^{0 \prime}(\sigma)=\frac{1}{\pi} \int_{0}^{\infty} \frac{d k}{1+\exp \left\{\beta\left(k^{2}-\sigma\right)\right\}} \sim \frac{1}{\pi} \sigma^{1 / 2}
$$

and

$$
p^{0}(\sigma)=\frac{2}{\pi} \int_{0}^{\infty} \frac{k^{2} d k}{1+\exp \left\{\beta\left(k^{2}-\sigma\right)\right\}} \sim \frac{2}{3 \pi} \sigma^{3 / 2},
$$

to conclude that $\tilde{I}^{\mu}(\bar{\rho}, \varepsilon) \rightarrow \infty$. This shows that the level sets $K_{b}=\left\{(\bar{\rho}, \varepsilon) \mid \tilde{I}^{\mu}(\bar{\rho}, \varepsilon) \leqq b\right\}$ are bounded and therefore compact. We can actually determine the essential domain [14] of $\tilde{I}^{\mu}$ :

Lemma 4.1. The essential domain of $\tilde{I}^{\mu}$ is the set

$$
D\left(\tilde{I}^{\mu}\right)=\left\{(\bar{\rho}, \varepsilon) \in \mathbf{R}^{2} \mid \bar{\rho} \geqq 0 ; \varepsilon \geqq \frac{1}{3} \pi^{2} \bar{\rho}^{3}\right\} .
$$

Proof. We first note that, if $\bar{\rho}<0$ or $\varepsilon<0$ then $\widetilde{I}^{\mu}(\bar{\rho}, \varepsilon)=\infty$, because we can take $\alpha \rightarrow-\infty$ respectively $x \rightarrow-\infty$. Now put $t=\beta k^{2}$ and $\tau=\beta \sigma$, and change variables 
in the integrals (4.6) and (4.7). We obtain

$$
\begin{aligned}
p^{0}(\sigma) & =\frac{1}{\pi} \beta^{-3 / 2} \int_{0}^{\infty} \frac{t^{1 / 2} d t}{1+e^{t-\tau}} \\
& =\frac{2}{3 \pi} \beta^{-3 / 2} \int_{0}^{\infty} \frac{t^{3 / 2} d t}{\left(1+e^{t-\tau}\right)\left(1+e^{\tau-t}\right)}
\end{aligned}
$$

and

$$
p^{0 \prime}(\sigma)=\frac{1}{\pi} \beta^{-1 / 2} \int_{0}^{\infty} \frac{t^{1 / 2} d t}{\left(1+e^{t-\tau}\right)\left(1+e^{\tau-t}\right)}
$$

Since

$$
\int_{0}^{\infty} \frac{d t}{\left(1+e^{t-\tau}\right)\left(1+e^{\tau-t}\right)}=\frac{1}{2}\left(1+\tanh \frac{1}{2} \tau\right)
$$

we can use Hölder's inequality to find

$$
p^{0 \prime}(\sigma) \leqq \frac{1}{\pi}\left(\frac{3 \pi}{2} p^{0}(\sigma)\right)^{1 / 3}\left\{\frac{1}{2}\left(1+\tanh \frac{1}{2} \tau\right)\right\}^{2 / 3}<\frac{1}{\pi}\left(\frac{3 \pi}{2} p^{0}(\sigma)\right)^{1 / 3}
$$

This means that (4.4) has a solution only if

$$
\varepsilon>\frac{1}{3} \pi^{2} \bar{\rho}^{3} \text {. }
$$

Suppose now that $\varepsilon<\frac{1}{3} \pi^{2} \bar{\rho}^{3}$. Then we can optimise in the $\alpha$-direction by solving

$$
p^{0 \prime}(\sigma)=\bar{\rho}(1-x)^{1 / 2} \text {. }
$$

The derivative in the $x$-direction is then negative so that the supremum is attained as $x \rightarrow-\infty$ and $\sigma \rightarrow \infty$ satisfying (4.13). Since the derivative is bounded by a strictly negative number, it follows that $\widetilde{I}^{\mu}(\bar{\rho}, \varepsilon)=\infty$.

We still have to consider the boundaries. If $\bar{\rho}=0$ then we can optimise in the $\alpha$-direction by taking $\alpha \rightarrow-\infty$. Then taking $x \rightarrow 1$ we find

$$
\tilde{I}^{\mu}(0, \varepsilon)=\varepsilon+p^{0}(\mu) \text {. }
$$

For the case $\varepsilon=\frac{1}{3} \pi^{2} \bar{\rho}^{3}$ we need the second terms in the asymptotic expansions for $p^{0}(\sigma)$ and $p^{0 \prime}(\sigma)$ :

$$
\left\{\begin{array}{l}
p^{0}(\sigma) \sim \frac{2}{3 \pi} \sigma^{3 / 2}+\frac{1}{12} \pi \beta^{-2} \sigma^{-1 / 2}+\cdots \\
p^{0 \prime}(\sigma) \sim \frac{1}{\pi} \sigma^{1 / 2}-\frac{1}{24} \pi \beta^{-2} \sigma^{-3 / 2}+\cdots
\end{array} .\right.
$$

We conclude that along the curve (4.13),

$$
\begin{aligned}
\varepsilon-\frac{1}{2}(1-x)^{-3 / 2} p^{0}(\sigma) & =\frac{1}{3} \pi^{2} \bar{\rho}^{3}-\frac{1}{2} \bar{\rho}^{3} \frac{p^{0}(\sigma)}{p^{0 \prime}(\sigma)^{3}} \\
& =\frac{1}{2} \bar{\rho}^{3}\left\{\frac{2}{3} \pi^{2}-\frac{p^{0}(\sigma)}{p^{0 \prime}(\sigma)^{3}}\right\} \\
& \approx-\frac{1}{12} \pi^{4} \bar{\rho}^{3} \beta^{-2} \sigma^{-2} .
\end{aligned}
$$


Since the integral $\int_{1}^{\infty} \sigma^{-2} d \sigma$ converges we conclude that $\tilde{I}^{\mu}\left(\bar{\rho}, \frac{1}{3} \pi^{2} \bar{\rho}^{-3}\right)<\infty$.

Let us now proceed with the proof of Theorem 4.1. It remains to show that LD. 3 and LD.4 are satisfied. (See Sect. 1.) This is standard: see e.g. Ellis [15] or [16]. In the proof of LD.3 one uses the following

Lemma 4.2. (Ellis [15]). Given $(\alpha, x) \in \mathbf{R}^{2}$ with $x<1$ and $\gamma \in \mathbf{R}$ define $H_{+}(\alpha, x, \gamma)$ by

$$
H_{+}(\alpha, x, \gamma)=\left\{(\bar{\rho}, \varepsilon) \in[0, \infty) \times[0, \infty) \mid \alpha \bar{\rho}+x \varepsilon-\widetilde{C}^{\mu}(\alpha, x) \geqq \gamma\right\} .
$$

If $C$ is a closed subset of $\mathbf{R}^{3}$ such that $\gamma<\tilde{I}^{\mu}(C)$ then there exists a finite set $\left(\alpha_{1}, x_{1}\right), \ldots,\left(\alpha_{r}, x_{r}\right)$ such that $C \subset \bigcup_{j=1}^{r} H_{+}\left(\alpha_{j}, x_{j}, \gamma\right)$.

Using Markov's inequality we find

$$
\begin{aligned}
\tilde{\mathbf{K}}_{l}^{\mu}[C] & \leqq \sum_{j=1}^{r} \tilde{\mathbf{K}}_{l}^{\mu}\left[H_{+}\left(\alpha_{j}, x_{j}, \gamma\right)\right] \\
& \leqq \sum_{j=1}^{r} e^{-\beta V_{l}\left\{\tilde{C}^{\mu}\left(\alpha_{j}, x_{j}\right)+\gamma\right\}} \int_{0}^{\infty} \int_{0}^{\infty} e^{\beta V_{l}\left(\alpha_{j} \bar{\rho}+x_{j} \varepsilon\right)} \widetilde{\mathbf{K}}_{l}^{\mu}(d \bar{\rho}, d \varepsilon) \\
& =\sum_{j=1}^{r} \exp \left[-\beta V_{l}\left\{\tilde{C}^{\mu}\left(\alpha_{j}, x_{j}\right)+\gamma-\tilde{C}_{l}^{\mu}\left(\alpha_{j}, x_{j}\right)\right\}\right]
\end{aligned}
$$

so that

$$
\limsup _{l \rightarrow \infty} \frac{1}{\beta V_{l}} \ln \tilde{\mathbf{K}}_{l}^{\mu}[C] \leqq-\gamma .
$$

But $\gamma<\tilde{I}^{\mu}[C]$ was arbitrary; hence

$$
\limsup _{l \rightarrow \infty} \frac{1}{\beta V_{l}} \ln \tilde{\mathbf{K}}_{l}^{\mu}[C] \leqq-\tilde{I}^{\mu}[C],
$$

which is LD.3.

It remains to prove LD.4: for any open set $O \subset[0, \infty) \times[0, \infty)$,

$$
\liminf _{l \rightarrow \infty} \frac{1}{\beta V_{l}} \ln \tilde{\mathbf{K}}_{l}^{\mu}[O] \geqq-\tilde{I}^{\mu}[O] .
$$

This is trivial if $O \subset D\left(\widetilde{I}^{\mu}\right)^{c}$. If $O \cap D\left(\widetilde{I}^{\mu}\right) \neq \emptyset$ then there exists a point $\left(\bar{\rho}_{0}, \varepsilon_{0}\right) \in G$, where $G=\operatorname{int}\left(O \cap D\left(\tilde{I}^{\mu}\right)\right)$ such that $\tilde{I}^{\mu}\left(\bar{\rho}_{0}, \varepsilon_{0}\right)<\widetilde{I}^{\mu}[O]+\delta$.

Let $\left(\alpha_{0}, x_{0}\right)$ be the corresponding solution of (4.4) and define the shifted measures

$$
\tilde{\mathbf{K}}_{0, l}^{\mu}(d \bar{\rho}, d \varepsilon)=\exp \left\{\beta V_{l}\left(\alpha_{0} \bar{\rho}+x_{0} \varepsilon-\tilde{C}_{l}^{\mu}\left(\alpha_{0}, x_{0}\right)\right\} \tilde{\mathbf{K}}_{l}^{\mu}(d \bar{\rho}, d \varepsilon)\right.
$$

Lemma 4.3. For l sufficiently large, $\tilde{\mathbf{K}}_{0, l}^{\mu}\left[B_{\delta}\right]>\frac{1}{2}$, where

$$
B_{\delta}=G \cap\left\{(\bar{\rho}, \varepsilon)|| \alpha_{0}\left(\bar{\rho}-\bar{\rho}_{0}\right)+x_{0}\left(\varepsilon-\varepsilon_{0}\right) \mid<\delta\right\} .
$$

Given this lemma we have

$$
\begin{aligned}
\tilde{\mathbf{K}}_{l}^{\mu}\left[B_{\delta}\right] & =\exp \left\{\beta V_{l} \tilde{C}_{l}^{\mu}\left(\alpha_{0}, x_{0}\right)\right\} \int_{B_{\delta}} \exp \left\{-\beta V_{l}\left(\alpha_{0} \bar{\rho}+x_{0} \varepsilon\right)\right\} d \tilde{\mathbf{K}}_{0, l}^{\mu}(d \bar{\rho}, d \varepsilon) \\
& \geqq \frac{1}{2} \exp \left\{\beta V_{l}\left(\tilde{C}_{l}^{\mu}\left(\alpha_{0}, x_{0}\right)-\alpha_{0} \bar{\rho}_{0}-x_{0} \varepsilon_{0}-\delta\right)\right\},
\end{aligned}
$$


so that

$$
\begin{aligned}
\liminf _{l \rightarrow \infty} \frac{1}{\beta V_{l}} \ln \tilde{\mathbf{K}}_{l}^{\mu}[O] & \geqq \widetilde{C}^{\mu}\left(\alpha_{0}, x_{0}\right)-\alpha_{0} \bar{\rho}_{0}-x_{0} \varepsilon_{0}-\delta \\
& =-\widetilde{I}^{\mu}\left(\bar{\rho}_{0}, \varepsilon_{0}\right)-\delta \geqq-\tilde{I}^{\mu}[O]-2 \delta
\end{aligned}
$$

This proves LD.4 since $\delta>0$ was arbitrary.

Proof of Lemma 4.3. We calculate the Laplace transform of $\tilde{\mathbf{K}}_{0, l}^{\mu}$ :

$$
\begin{aligned}
& \int e^{-s \bar{\rho}-t \varepsilon} \tilde{\mathbf{K}}_{0, l}^{\mu}(d \bar{\rho}, d \varepsilon) \\
& \quad=\exp \left\{-\beta V_{l} \tilde{C}_{l}^{\mu}\left(\alpha_{0}, x_{0}\right)\right\} \int \exp \left\{\beta V_{l}\left(\left(\alpha_{0}-\frac{s}{\beta V_{l}}\right) \bar{\rho}+\left(x_{0}-\frac{t}{\beta V_{l}}\right) \varepsilon\right)\right\} \tilde{\mathbf{K}}_{l}^{\mu}(d \bar{\rho}, d \varepsilon) \\
& \quad=\exp \left\{-\beta V_{l} \tilde{C}_{l}^{\mu}\left(\alpha_{0}, x_{0}\right)+\beta V_{l} \tilde{C}_{l}^{\mu}\left(\alpha_{0}-\frac{s}{\beta V_{l}}, x_{0}-\frac{t}{\beta V_{l}}\right)\right\} \\
& \quad \rightarrow \exp \left\{-s \frac{\partial \tilde{C}^{\mu}}{\partial \alpha}\left(\alpha_{0}, x_{0}\right)-t \frac{\partial \tilde{C}^{\mu}}{\partial x}\left(\alpha_{0}, x_{0}\right)\right\} \\
& \quad=\exp \left\{-s \bar{\rho}_{0}-t \varepsilon_{0}\right\} .
\end{aligned}
$$

This means that $\tilde{\mathbf{K}}_{0, l}^{\mu} \rightarrow \delta_{\left(\bar{\rho}_{0}, \varepsilon_{0}\right)}$ as $l \rightarrow \infty$.

The proof of the large deviation principle for the second-level measures $\left\{\mathbf{K}_{l}^{\mu}\right\}$ is very similar:

Theorem 4.2. The sequence $\left\{\mathbf{K}_{l}^{\mu} \mid l=1,2, \ldots\right\}$ of probability measures on $E$ defined by (2.12) satisfies the large deviation property with constants $\beta V_{l}$ and rate function $I^{\mu}$ given by (3.1).

Proof. The lower semicontinuity of $I^{\mu}$ follows from the fact that it is a Legendre transform. Since the topology on $E$ is the weak-* topology, LD.2 follows if we can prove boundedness of the level sets $K_{b}=\left\{m \mid I^{\mu}[m] \leqq b\right\}$. But if $I^{\mu}[m] \leqq b$ then $\langle t, m\rangle \leqq C^{\mu}[t]+b$ for every $t \in F$ and therefore $|\langle t, m\rangle| \leqq C^{\mu}[|t|]+b$, which proves that $K_{b}$ is weak-* bounded and hence bounded. In the infinite-dimensional case Lemma 4.2 is not valid, but the corresponding statement for compact sets is true:

Lemma 4.4. Let $K \subset E$ be compact. Given $t \in F$ and $\gamma \in \mathbf{R}$ we define

$$
H_{+}(t, \gamma)=\left\{m \in E \mid\langle t, m\rangle-C^{\mu}[t] \geqq \gamma\right\} \text {. }
$$

If $\gamma<I^{\mu}[K]$, then there exists a finite set $t_{1}, \ldots, t_{r} \in F$ such that $K \subset \bigcup_{j=1}^{r} H_{+}\left(t_{j}, \gamma\right)$.

The upper bound

$$
\limsup _{l \rightarrow \infty} \frac{1}{\beta V_{l}} \ln \mathbf{K}_{l}^{\mu}[K] \leqq-I^{\mu}[K]
$$

then follows as before. If $C \subset E$ is a general closed set we can make use of the large deviation result, Theorem 4.1 above. Indeed, the sets

$$
B_{R}=\left\{m \in E \mid \int\left(1+k^{2}\right) m(d k) \leqq R\right\}
$$


are compact subsets in $E$ so that the upper bound holds for $C \cap B_{R}$. But

Therefore,

$$
\mathbf{K}_{l}^{\mu}\left[B_{R}^{c}\right] \leqq \tilde{\mathbf{K}}_{l}^{\mu}[(\bar{\rho}, \varepsilon) \mid \bar{\rho}+\varepsilon \geqq R] \leqq-\inf _{\bar{\rho}+\varepsilon \geqq R} \tilde{I}^{\mu}(\bar{\rho}, \varepsilon) \rightarrow-\infty \quad \text { as } \quad R \rightarrow \infty .
$$

$$
\begin{aligned}
\limsup _{l \rightarrow \infty} \frac{1}{\beta V_{l}} \ln \tilde{\mathbf{K}}_{l}^{\mu}[C] & \leqq \limsup _{l \rightarrow \infty} \frac{1}{\beta V_{l}} \ln \left\{\tilde{\mathbf{K}}_{l}^{\mu}\left[C \cap B_{R}\right]+\tilde{\mathbf{K}}_{l}^{\mu}\left[B_{R}^{c}\right]\right\} \\
& \leqq \limsup _{l \rightarrow \infty} \frac{1}{\beta V_{l}} \ln \tilde{\mathbf{K}}_{l}^{\mu}\left[C \cap B_{R}\right] \vee \limsup _{l \rightarrow \infty} \frac{1}{\beta V_{l}} \ln \tilde{\mathbf{K}}_{l}^{\mu}\left[B_{R}^{c}\right] \\
& =\limsup _{l \rightarrow \infty} \frac{1}{\beta V_{l}} \ln \tilde{\mathbf{K}}_{l}^{\mu}\left[C \cap B_{R}\right] \leqq-I^{\mu}\left[C \cap B_{R}\right] \leqq-I^{\mu}[C] .
\end{aligned}
$$

In the proof of LD. 4 we make use of the approximation theorem: Let $O \subset E$ be open. Then Theorem 3.2 says that, given $\delta>0$, there exists a Fermi-Dirac measure $m_{0}(d k)=\rho^{\mu+t_{0}}(k) d k / 2 \pi$ such that $m_{0} \in O$ and $I^{\mu}\left[m_{0}\right]<I^{\mu}[O]+\delta$. Again we define the shifted measures

$$
\mathbf{K}_{0, l}^{\mu}[d m]=\exp \left\{\beta V_{l}\left(\left\langle t_{0}, m\right\rangle-C_{l}^{\mu}\left[t_{0}\right]\right)\right\} \mathbf{K}_{l}^{\mu}[d m],
$$

and prove

Lemma 4.5. For l sufficiently large, $\mathbf{K}_{0, l}^{\mu}\left[B_{\delta}\right]>\frac{1}{2}$, where

$$
B_{\delta}=O \cap\left\{m \in E||\left\langle m-m_{0}, t_{0}\right\rangle \mid<\delta\right\} .
$$

Given this lemma the proof proceeds as in the proof of Theorem 4.1.

Proof of Lemma 4.5. Since $B_{\delta}$ is open, there exists a finite set $t_{1}, \ldots, t_{r} \in F$ such that

$$
U_{\delta} \equiv\left\{m \in E||\left\langle t_{i}, m-m_{0}\right\rangle \mid<\delta \text { for } i=1,2, \ldots, r\right\} \subset B_{\delta} .
$$

Let $\mathbf{Q}_{l}$ be the marginal distribution of the variables $\left\langle t_{1}, m\right\rangle, \ldots,\left\langle t_{r}, m\right\rangle$. We compute its Laplace transform as in the proof of Lemma 4.3:

$$
\begin{aligned}
\int e^{-\left(s_{1} u_{1}+\cdots+s_{r} u_{r}\right)} \mathbf{Q}_{l}\left(d u_{1}, \ldots, d u_{r}\right) & =\exp \left\{\beta V_{l}\left(C_{l}^{\mu}\left[t_{0}-\frac{1}{\beta V_{l}} \sum_{i=1}^{r} s_{i} t_{i}\right]-C_{l}^{\mu}\left[t_{0}\right]\right)\right\} \\
& \rightarrow \exp \left\{\left.\sum_{i=1}^{r} s_{i} \frac{d}{d s}\right|_{s=0} C^{\mu}\left[t_{0}+s t_{i}\right]\right\} \\
& =\exp \left\{-\sum_{i=1}^{r} s_{i}\left\langle t_{i}, m_{0}\right\rangle\right\} .
\end{aligned}
$$

Thus $\mathbf{Q}_{l}$ converges to the $\delta$-measure on $\left(\left\langle t_{1}, m_{0}\right\rangle, \ldots,\left\langle t_{r}, m_{0}\right\rangle\right)$. It follows that, with $u_{i}^{0}=\left\langle t_{i}, m_{0}\right\rangle$,

$$
\mathbf{K}_{l}^{\mu}\left[B_{\delta}\right] \geqq \mathbf{K}\left[U_{\delta}\right]=\mathbf{Q}_{l}\left[\left|u_{i}-u_{i}^{0}\right|<\delta\right] \rightarrow 1 .
$$

\section{Existence of the Finite-Volume Pressure}

After all the preliminary work in Sects. 2-4 we can finally start considering the interacting model. As explained in the introduction the $N$-particle Hamiltonian 
(1.12) can be diagonalised with the help of the Bethe Ansatz, and the eigenvalues can be labelled by the sets $\left\{k_{1}, \ldots, k_{N}\right\}$ of distinct momenta $k_{j}=\left(2 \pi / V_{l}\right) I_{j}$ with $I_{j}=n_{j}$ if $N$ is odd, and $I_{j}=n_{j}+\frac{1}{2}$ if $N$ is even. We have seen that, assuming the completeness of the Bethe Ansatz eigenstates, the eigenvalues are given by

$$
E\left(\left\{k_{1}, \ldots, k_{N}\right\}\right)=\sum_{j=1}^{N} \tilde{k}_{j}\left(k_{1}, \ldots, k_{N}\right)^{2}
$$

where the $\tilde{k}_{j}\left(k_{1}, \ldots, k_{N}\right)$ are defined as follows.

Define, for any measure $m \in E$, the function $f_{m}: \mathbf{R} \rightarrow \mathbf{R}$ as the unique solution of

$$
f_{m}(k)=k-\int \theta_{c}\left(f_{m}(k)-f_{m}\left(k^{\prime}\right)\right) m\left(d k^{\prime}\right) .
$$

Let $m_{l}$ be the occupation measure,

$$
m_{l}[A ; \sigma]=\frac{1}{V_{l}} \sum_{n \in \mathbf{Z}} \sigma_{n} \delta_{k_{n}^{l}(\sigma)}[A]
$$

with $\sigma_{n_{j}}=1(j=1,2, \ldots, N)$ and $\sigma_{n}=0$ if $n \neq n_{j}$ for any $j$. The $k_{n}^{l}(\sigma)$ are defined by (1.24). Clearly $k_{n_{j}}^{l}(\sigma)=k_{j}$. Given all this, $\tilde{k}_{j}$ is defined as

$$
\tilde{k}_{j}=f_{m_{l}}\left(k_{j}\right) \text {. }
$$

Presently we show that $f_{m}$ exists and is unique. First we establish this in the $L^{2}$-space:

Proposition 5.1. Let $m \in E$. Then there exists a unique solution $f_{m} \in L^{2}(\mathbf{R}, d m)$ to (5.2).

Proof. This is a rigorous version of the argument of Yang \& Yang [1]. We define a functional $B: L^{2}(\mathbf{R}, d m) \rightarrow \mathbf{R}$ by

$$
B[f]=\frac{1}{2} \int f(k)^{2} m(d k)-\int k f(k) m(d k)+\frac{1}{2} \iint \Theta_{c}\left(f(k)-f\left(k^{\prime}\right)\right) m(d k) m\left(d k^{\prime}\right),
$$

where

$$
\Theta_{c}(k)=\int_{0}^{k} \theta_{c}\left(k^{\prime}\right) d k^{\prime}
$$

We calculate the Gateaux derivative:

$$
\begin{aligned}
D B[f] g= & \int f(k) g(k) m(d k)-\int k g(k) m(d k) \\
& +\iint \theta_{c}\left(f(k)-f\left(k^{\prime}\right)\right)\left(g(k)-g\left(k^{\prime}\right)\right) m(d k) m\left(d k^{\prime}\right) \\
= & \int\left\{f(k)-k+\int \theta_{c}\left(f(k)-f\left(k^{\prime}\right)\right) m\left(d k^{\prime}\right)\right\} g(k) m(d k) .
\end{aligned}
$$

Since $g \in L^{2}(\mathbf{R}, d m)$ is arbitrary, we find that

$$
D B[f]=0 \Leftrightarrow f(k)=k-\int \theta_{c}\left(f(k)-f\left(k^{\prime}\right)\right) m\left(d k^{\prime}\right) \text { for } m \text {-a.e. } k .
$$

A simple calculation shows that $B$ is strictly convex. Furthermore, using the fact that $\left|\Theta_{c}(k)\right| \leqq \pi|k|$, we have

$$
B[f] \geqq \frac{1}{2}\|f\|^{2}-\|f\|\left(\int k^{2} m(d k)\right)^{1 / 2}-\pi\|f\|\|m\|^{3 / 2},
$$

so that $B[f]$ is bounded below. It also follows from (5.9) that $B[f] \rightarrow \infty$ as $\|f\| \rightarrow \infty$. We can now apply Theorem 1.2 of Barbu \& Precupanu [14] to conclude 
that $B[f]$ attains a unique minimum at $f_{m} \in L^{2}(\mathbf{R}, d m)$. (See also Remark 1.1 and Remark 1.2 below Theorem 1.2 in [14].) The minimiser $f_{m}$ clearly satisfies (5.8).

Proposition 5.2. Let $m \in$ E. Equation (5.2) has a unique solution $f_{m} \in \mathscr{C}^{\infty}(\mathbf{R}) \cap \mathscr{L}^{2}(\mathbf{R}$. $(l n)$. Proof. Let $\tilde{f}_{m} \in \mathscr{L}^{2}(\mathbf{R}, d m)$ be a solution of $(5.2)$ for $m$-a.e. $k$. Then $\tilde{f}_{m}$ is $m$-measurable and the image measure $\tilde{m}=\tilde{f}_{m}(m)$ is well-defined. Now define $h_{\tilde{m}}: \mathbf{R} \rightarrow \mathbf{R}$ by

$$
h_{\tilde{m}}(k)=k+\int \theta_{c}\left(k-k^{\prime}\right) \tilde{m}\left(d k^{\prime}\right) .
$$

Clearly $h \in \mathscr{C}^{\infty}$ and $h^{\prime}(k)>1$. Hence $h$ is invertible. Let $f_{m}$ be the inverse. Then $f_{m} \in \mathscr{C}^{\infty}$ and $f_{m}$ satisfies (5.2) for all $k$. This equation implies

$$
\left|f_{m}(k)-k\right| \leqq \pi\|m\|
$$

so that $f_{m} \in \mathscr{L}^{2}(\mathbf{R}, d m)$.

Given the solution $f_{m_{l}}$ the eigenvalues (5.1) are fully determined and the expression (1.1) for the finite-volume pressure $p_{l}(\mu)$ becomes

$$
\exp \left\{\beta V_{l} p_{l}(\mu)\right\}=\sum_{N=0}^{\infty} e^{\beta \mu N} \sum_{\underline{k} \in \mathscr{P}_{l}^{N}} e^{-\beta E(\underline{k})}
$$

where

$\mathscr{P}_{l}^{N}=\left\{\underline{k}=\left\{k_{1}, \ldots, k_{N}\right\} \mid k_{j} \in \frac{2 \pi}{V_{l}} \mathbf{Z}\right.$ if $N$ is odd, and $k_{j} \in \frac{2 \pi}{V_{l}}\left(\mathbf{Z}+\frac{1}{2}\right)$ if $N$ is even $\}$.

Warning: $\left\{k_{1}, \ldots, k_{N}\right\}$ is meant in the sense of sets, i.e. it is an unordered $N$-tuple of distinct $k_{j}$ 's.

Presently we show that the series in (5.12) converges, so that the finite-volume pressure is well-defined. We write (5.12) as follows:

$$
\exp \left\{\beta V_{l} p_{l}(\mu)\right\}=\sum_{N=0}^{\infty} e^{\beta \mu N} \sum_{\left\{\sigma \in \Omega \mid \sum \sigma_{n}=N\right\}} \exp \left\{-\beta \sum_{n \in \mathbf{Z}} \sigma_{n} f_{m_{l}}\left(k_{m}^{l}(\sigma)\right)^{2}\right\} .
$$

We need an estimate on the function $f_{m_{l}}(k)$. Differentiating the defining relation (5.2) we obtain

$$
f_{m}^{\prime}(k)=\left[1+\int \theta_{c}^{\prime}\left(f_{m}(k)-f_{m}\left(k^{\prime}\right)\right) m\left(d k^{\prime}\right)\right]^{-1} .
$$

A very simple estimate can be obtained in the following fashion. Since

$$
\theta_{c}^{\prime}(k)=\frac{2 c}{c^{2}+k^{2}} \leqq \frac{2}{c}
$$

we have

$$
1 \geqq f^{\prime}(k) \geqq \frac{1}{1+2 c^{-1} \bar{\rho}} \quad(\bar{\rho}=\|m\|) .
$$

We order the set $\left\{k_{j}\right\}$ so that $k_{j}<k_{j+1}$ and define $\Delta k_{j}=k_{j+1}-k_{j}$. The set 
$\left\{\tilde{k}_{j} \mid j=1, \ldots, N\right\}$ is then also ordered since $\tilde{k}_{j}=f_{m_{l}}\left(k_{j}\right)$ and $f_{m_{l}}$ is increasing. Putting $\Delta \widetilde{k}_{j}=\tilde{k}_{j+1}-\tilde{k}_{j}$ we conclude from (5.17) that

$$
\Delta \tilde{k}_{j} \geqq \alpha \Delta k_{j} \quad \text { with } \quad \alpha=\frac{1}{1+2 c^{-1} \bar{\rho}} .
$$

We can then use

Lemma 5.1 If $\Delta \tilde{k}_{j} \geqq \alpha \Delta k_{j}$, then

$$
\sum_{j=1}^{N}\left(\tilde{k}_{j}-\frac{1}{N} \sum_{i=1}^{N} \tilde{k}_{i}\right)^{2} \geqq \alpha^{2} \sum_{j=1}^{N}\left(k_{j}-\frac{1}{N} \sum_{i=1}^{N} k_{i}\right)^{2} .
$$

Proof. Let $\Delta_{j}=\Delta k_{j} ; \tilde{\Delta}_{j}=\Delta \tilde{k}_{j}$. We have

$$
\sum_{j=1}^{N}\left(k_{j}-\frac{1}{N} \sum_{i=1}^{N} k_{i}\right)^{2}=\frac{1}{N^{2}} \sum_{i=1}^{N}\left(\sum_{j=1}^{i-1} j \Delta_{j}-\sum_{j=i}^{N-1}(N-j) \Delta_{j}\right)^{2} .
$$

This is a homogeneous, second order expression in the $\Delta_{j}(j=1, \ldots, N-1)$. The coefficient of $\Delta_{j}^{2}$ is $N j(N-j)$ and the coefficient of $\Delta_{i} \Delta_{j}(i<j)$ is $2 i\left(N^{2}-N j-j\right)>0$. All coefficients are therefore positive and (5.19) follows.

We also have, from (5.2),

$$
\int f_{m}(k) m(d k)=\int k m(d k)
$$

which in the case $m=m_{l}$, reads

$$
\sum_{j=1}^{N} \tilde{k}_{j}=\sum_{j=1}^{N} k_{j}
$$

Together with Lemma 5.1 this implies that

$$
\sum_{j=1}^{N} \tilde{k}_{j}^{2} \geqq \alpha^{2} \sum_{j=1}^{N} k_{j}^{2}
$$

For fixed $N, \bar{\rho}$ and hence $\alpha$ is fixed. Thus (5.22) gives

$$
\sum_{\underline{k} \in \mathscr{P}_{l}^{N}} \exp \left\{-\beta \sum_{j=1}^{N} \tilde{k}_{j}^{2}\right\} \leqq \sum_{\underline{k} \in \mathscr{P}_{l}^{N}} \exp \left\{-\beta \alpha^{2} \sum_{j=1}^{N} k_{j}^{2}\right\}<\infty .
$$

However, the bound (5.22) is not sufficient to ensure that convergence of the sum over $N$ in (5.12). This fact can be appreciated by restricting the sum in (5.23) to the ground state:

$$
k_{j}^{0}=\frac{2 \pi}{V_{l}}\left(-\frac{N+1}{2}+j\right) \quad(j=1, \ldots, N) .
$$

Then $\sum_{j=1}^{N} k_{j}=\mathcal{O}\left(N^{3}\right)$, while $\alpha=\mathcal{O}\left(N^{-1}\right)$ so that

$$
\sum_{N=0}^{\infty} e^{\beta \mu N} \sum_{\underline{k} \in \mathscr{P}_{l}^{N}} \exp \left\{-\beta \alpha^{2} \sum_{j=1}^{N} k_{j}^{2}\right\} \geqq \sum_{N=0}^{\infty} e^{\beta \mu N} \cdot e^{-\beta \mathcal{O}(N)} .
$$

This expression diverges for large $\mu$. 
We can improve on the bound (5.22) by the following iterative procedure. We reinsert (5.18) into (5.15).:

$$
f_{m_{l}}^{\prime}(k)=\left[1+\frac{2 c}{V_{l}} \sum_{i=1}^{N} \frac{1}{c^{2}+\left(\tilde{k}-\tilde{k}_{j}\right)^{2}}\right]^{-1} \geqq\left[1+\frac{2 c}{V_{l}} \sum_{i=1}^{N} \frac{1}{c^{2}+\alpha^{2}\left(k-k_{i}\right)^{2}}\right]^{-1} .
$$

Next we show that the sum in this expression can be bounded by the corresponding ground state sum:

Lemma 5.2. We have

$$
\sum_{i=1}^{N}\left\{c^{2}+\alpha^{2}\left(k-k_{i}\right)^{2}\right\}^{-1} \leqq \sum_{j=-(N-1) / 2}^{(N-1) / 2}\left\{c^{2}+\left(\frac{2 \pi \alpha}{V_{l}}\right)^{2} j^{2}\right\}^{-1}
$$

provided that $V_{l}$ is large enough.

Proof. By shifting over multiples of $2 \pi / V_{l}$ it is clear that it is sufficient to prove this inequality in the case that $0 \leqq k<\left(2 \pi / V_{l}\right)$. Now let $j$ be the index such that $k_{j} \leqq 0$ and $k_{j+1} \geqq\left(2 \pi / V_{l}\right)\left(j=0\right.$ if $k_{1} \geqq\left(2 \pi / V_{l}\right)$ and $j=N+1$ if $\left.k_{N} \leqq 0\right)$. We push the $k_{i}$ with $i \leqq j$ to the right and the $k_{i}$ with $i \geqq j+1$ to the left to conclude that

$$
\begin{aligned}
\sum_{i=1}^{N}\left\{c^{2}+\alpha^{2}\left(k-k_{i}\right)^{2}\right\}^{-1} \leqq & \sum_{i=1}^{j}\left\{c^{2}+\alpha^{2}\left(k+\frac{2 \pi}{V_{l}}(j-i)\right)^{2}\right\}^{-1} \\
& +\sum_{i=j+1}^{N}\left\{c^{2}+\alpha^{2}\left(\frac{2 \pi}{V_{l}}(i-j)-k\right)^{2}\right\}^{-1} .
\end{aligned}
$$

The lemma now follows from Lemma 5.3.

Lemma 5.3. There exists a constant $A$ such that, for $a>A$,

$$
\sum_{j=-(N-1) / 2}^{(N-1) / 2} \frac{1}{a^{2}+(p-j)^{2}} \leqq \sum_{j=-(N-1) / 2}^{(N-1) / 2} \frac{1}{a^{2}+j^{2}}
$$

for all positive integers $N$, and $p \in \mathbf{R}$.

Proof. By symmetry we may assume that $0 \leqq p<\frac{1}{2}$. We calculate the derivative and distinguish between $N$ odd or even. If $N=2 m+1$ is odd we write

$$
\begin{aligned}
& \frac{\partial}{\partial p_{j}} \sum_{-m}^{m} \frac{1}{a^{2}+(p-j)^{2}}=\frac{-2 p}{\left(a^{2}+p^{2}\right)^{2}}+\sum_{j=1}^{m}\left\{\frac{-2(p-j)}{\left[a^{2}+(p-j)^{2}\right]^{2}}+\frac{-2(p+j)}{\left[a^{2}+(p+j)^{2}\right]^{2}}\right\} \\
& \quad=-\frac{2 p}{\left(a^{2}+p^{2}\right)^{2}}-4 p \sum_{j=1}^{m} \frac{a^{4}+2\left(p^{2}-j^{2}\right) a^{2}+\left(p^{2}-j^{2}\right)\left(p^{2}+3 j^{2}\right)}{\left[a^{2}+(p-j)^{2}\right]^{2}\left[a^{2}+(p+j)^{2}\right]^{2}} .
\end{aligned}
$$

When $N=2 m$ is even,

$$
\frac{\partial}{\partial p} \sum_{j=-m+(1 / 2)}^{m-(1 / 2)} \frac{1}{a^{2}+(p-j)^{2}}=-4 p \sum_{j=1 / 2}^{m-(1 / 2)} \frac{a^{4}+2\left(p^{2}-j^{2}\right) a^{2}+\left(p^{2}-j^{2}\right)\left(p^{2}+3 j^{2}\right)}{\left[a^{2}+(p-j)^{2}\right]^{2}\left[a^{2}+(p+j)^{2}\right]^{2}} .
$$

In both cases it is sufficient to prove that the sum in the final expression is positive 
irrespective of $m$. Since $0 \leqq p<\frac{1}{2}$ these sums are bounded by

$$
\begin{aligned}
& \sum_{j=1 \text { resp. (1/2) }}^{\infty} \frac{a^{4}-2 j^{2} a^{2}-3 j^{4}}{\left[a^{2}+j^{2}\right]^{2}\left[a^{2}+\left(j+\frac{1}{2}\right)^{2}\right]^{2}} \\
& =\frac{1}{a^{3}} \sum_{j=1}^{\infty} \frac{1-3\left(\frac{j}{a}\right)^{2}}{1} \frac{1-(1 / 2)}{a\left(1+\left(\frac{j}{a}\right)^{2}\right)\left(1+\left(\frac{j+1 / 2}{a}\right)^{2}\right)^{2}} .
\end{aligned}
$$

As $a \rightarrow \infty$ this sum tends to

$$
\int_{0}^{\infty} \frac{1-3 x^{2}}{\left(1+x^{2}\right)^{3}} d x=\frac{7}{16} \pi>0
$$

We can bound the sum on the right-hand side of (5.26) by an integral:

If $N=2 m+1$ is odd,

$$
\sum_{j=-m}^{m} \frac{1}{a^{2}+j^{2}}<2 \int_{0}^{m} \frac{d x}{a^{2}+x^{2}}+\frac{1}{a^{2}},
$$

and if $N=2 m$ is even,

$$
\sum_{j=-m+(1 / 2)}^{m-(1 / 2)} \frac{1}{a^{2}+j^{2}}<2 \int_{0}^{m-(1 / 2)} \frac{d x}{a^{2}+x^{2}}+\frac{1}{a^{2}} .
$$

Therefore, in all cases,

$$
\sum_{i=1}^{N} \frac{1}{c^{2}+\alpha^{2}\left(k-k_{i}\right)^{2}}<\frac{V_{l}}{2 \pi \alpha c} \int_{-\pi \alpha \bar{\rho} / c}^{\pi \alpha \bar{\rho} / c} \frac{d x}{1+x^{2}}+\frac{1}{c^{2}} .
$$

Inserting this into the bound (5.25) for $f_{m_{l}}^{\prime}(k)$ we find an improved bound: $f_{m_{l}}^{\prime}(k) \geqq \alpha^{\prime}$, with

$$
\alpha^{\prime}=\left[1+\frac{2}{V_{l} c}+\frac{2}{\pi \alpha} \arctan \left(\frac{\pi \bar{\rho}}{c} \alpha\right)\right]^{-1} .
$$

Iterating the above procedure we obtain better and better bounds $f_{m_{l}}^{\prime}(k) \geqq \alpha^{(n)}$ with $\alpha^{(n)}=\alpha^{(n-1) /}$. As $n \rightarrow \infty, \alpha^{(n)}$ approaches a fixed point $\alpha^{*}$ of (5.28). Putting $u=(\pi \bar{\rho} / c) \alpha^{*}$ we have

$$
\frac{2}{\pi} \arctan u=1-\left(1+\frac{2}{V_{l} c}\right) \frac{c}{\pi \bar{\rho}} u .
$$

As $\bar{\rho} \rightarrow \infty, u \rightarrow \infty$ and asymptotically,

$$
1-\frac{2}{\pi u} \sim 1-\left(1+\frac{2}{V_{l} c}\right) \frac{c}{\pi \bar{\rho}} u \Rightarrow u^{2} \sim \frac{2 \bar{\rho}}{c}\left(1+\frac{2}{V_{l} c}\right)^{-1}>\frac{\bar{\rho}}{c} .
$$

From this we conclude that, for large $\bar{\rho}$,

$$
f_{m_{l}}^{\prime}(k)>\frac{1}{\pi}\left(\frac{c}{\bar{\rho}}\right)^{1 / 2}
$$


and with Lemma 5.1:

Lemma 5.5. For $\bar{\rho}$ large enough,

$$
\sum_{i=1}^{N} \tilde{k}_{i}^{2}>\frac{c}{\pi^{2}} \frac{\varepsilon}{\bar{\rho}}
$$

This bound is sufficient to ensure the convergence of the series (5.12):

Proposition 5.3. For all $\mu \in \mathbf{R}, p_{l}(\mu)<\infty$.

Proof. We write $L=V_{l}$ and $\kappa=\left(\beta c / \pi^{2}\right)$. Assume that the bound (5.31) holds for $N \geqq N_{0}$. We estimate the sum (5.23) in the case $N \geqq N_{0}$ :

$$
\begin{aligned}
\sum_{\underline{k}} \exp \left\{-\beta \sum_{i=1}^{N} \tilde{k}_{i}^{2}(\underline{k})\right\} & \leqq \sum_{\underline{k}} \exp \left\{-\kappa \frac{L}{N} \sum_{i=1}^{N} k_{i}^{2}\right\} \\
& =\sum_{\left\{n_{i}\right\}_{i=1}^{N}} \exp \left\{-\kappa \frac{L}{N}\left(\frac{2 \pi}{L}\right)^{2} \sum_{i=1}^{N} n_{i}^{2}\right\},
\end{aligned}
$$

where $n_{i} \in \mathbf{Z}$ if $N$ is odd, and $n_{i}+\frac{1}{2} \in \mathbf{Z}$ if $N$ is even. We now estimate $\Sigma n_{i}^{2}$ by splitting off the ground state:

\section{Lemma 5.6.}

$$
\sum_{i=1}^{N} n_{i}^{2} \geqq \sum_{i=-(N-1) / 2}^{(n-1) / 2} i^{2}+\sum_{i=1}^{N}\left(n_{i}-i+\frac{N+1}{2}\right)^{2} .
$$

Proof. By induction for odd and even $N$ separately. Consider odd $N$. The case $N=1$ is trivial. The induction step amounts to proving

$$
n_{1}^{2}+n_{N+2}^{2} \geqq 2\left(\frac{N+1}{2}\right)^{2}+\left(n_{1}+\frac{N+1}{2}\right)^{2}+\left(n_{N+2}-\frac{N+1}{2}\right)^{2} \text {. }
$$

This follows from $n_{N+2}-n_{1} \geqq N+1$.

Inserting (5.33) into (5.32) and using the fact that

$$
\sum_{i=-(N-1) / 2}^{(N-1) / 2} i^{2} \geqq \frac{1}{12}(N-1)^{3},
$$

we obtain, after summing over $N$ :

$$
\begin{aligned}
\sum_{N=N_{0}}^{\infty} e^{\beta \mu N} \sum_{\underline{k} \in \mathscr{P}_{l}^{N}} \exp \left\{-\beta \sum_{i=1}^{N} \tilde{k}_{i}^{2}(\underline{k})\right\} \leqq & \sum_{N=N_{0}}^{\infty} \exp \left\{\beta \mu N-\frac{\pi^{2}}{3 L} \kappa \frac{(N-1)^{3}}{N}\right\} \\
& \cdot \prod_{i=1}^{N} \sum_{m_{i} \in \mathbf{Z}} \exp \left\{-\frac{4 \pi^{2} \kappa}{L N} m_{i}^{2}\right\} .
\end{aligned}
$$

For $\alpha<\pi$ we have $\sum_{m=-\infty}^{\infty} e^{-\alpha m^{2}}<2 \sqrt{\pi / \alpha}$. Therefore 


$$
\begin{aligned}
& \sum_{N=N_{0}}^{\infty} e^{\beta \mu N} \sum_{\underline{k} \in \mathscr{P}_{l}^{N}} \exp \left\{-\beta \sum_{i=1}^{N} \tilde{k}_{i}^{2}(\underline{k})\right\} \\
& \leqq 2 \sum_{N=N_{0}}^{\infty}\left(\frac{N L}{\pi \kappa}\right)^{N / 2} \exp \left\{\beta \mu N-\frac{\pi^{2} \kappa}{3 L} \frac{(N-1)^{3}}{N}\right\}<\infty .
\end{aligned}
$$

This proves the proposition.

\section{Continuity of the Functional}

As explained in the introduction we can rewrite the expression (5.14) for the pressure $p_{l}(\mu)$ as an expectation with respect to the Kac measure $\mathbf{K}_{l}^{\mu}$ :

$$
\exp \left\{\beta V_{l} p_{l}(\mu)\right\}=\exp \left\{\beta V_{l} p_{l}^{0}(\mu)\right\} \int_{E} \exp \left\{\beta V_{l} G[m]\right\} \mathbf{K}_{l}^{\mu}[d m]
$$

with

$$
G[m]=\int\left(k^{2}-f_{m}(k)^{2}\right) m(d k) .
$$

There remain two obstacles to be overcome before we can apply Varadhan's Theorem. First we have to show that $G[m]$ is continuous, and second, that it satisfies condition (1.36). We shall deal with the latter problem in the next section, and prove the continuity of $G$ in the present section. We first consider the map $m \mapsto f_{m}$ and prove

Proposition 6.1. Let $F_{3 / 4}$ be the space

$$
F_{3 / 4}=\left\{f \in \mathscr{C}(\mathbf{R}) \mid f(k)=\left(1+k^{2}\right)^{3 / 4} \phi(k) \text { with } \phi \in \mathscr{C}_{0}(\mathbf{R})\right\}
$$

equipped with the norm

$$
\|f\|_{3 / 4}=\sup _{k \in \mathbf{R}}\left\{\left(1+k^{2}\right)^{-3 / 4}|f(k)|\right\} .
$$

For every $m \in E$ there exists a unique $f_{m} \in F_{3 / 4}$ satisfying (5.2), and the map $m \mapsto f_{m}$ is continuous: $E \rightarrow F_{3 / 4}$.

Proof. The bound (5.11) implies that the unique solution $f_{m} \in \mathscr{C}^{\infty}(\mathbf{R}) \cap \mathscr{L}^{2}(\mathbb{R}, d m)$ found in Proposition 5.2 belongs to $F_{3 / 4}$. Conversely, if $f \in F_{3 / 4}$ satisfies (5.2) then it obeys the bound (5.11) so that it is an element of $\mathscr{L}^{2}(\mathbf{R}, d m)$. It is therefore uniquely defined on the support of $m$ and hence everywhere by (5.2). Indeed, $y \mapsto k-\int \theta_{c}\left(y-f\left(k^{\prime}\right)\right) m\left(d k^{\prime}\right)$ is monotonically decreasing, so that the equation $y=k-\int \theta_{c}\left(y-f\left(k^{\prime}\right)\right) m\left(d k^{\prime}\right)$ has a unique solution $y=f(k)$.

Now consider a net $\left(m_{\alpha}\right)_{\alpha \in A}$ in $E$ converging to $m \in E$. We shall prove that $f_{m_{\alpha}}$ converges to $f_{m}$ using the following well-known lemma:

Lemma 6.1. Let $\left(x_{\alpha}\right)_{\alpha \in A}$ be a net in a topological space $X$ such that every subnet has a subnet converging to $x \in X$. Then $x_{\alpha}$ converges to $x$.

Let, therefore, $\left(m_{\beta}^{(1)}\right)_{\beta \in B}$ be a subnet of $\left(m_{\alpha}\right)$. It follows from the bound (5.11) that $\left\{f_{m_{\beta}^{(1)}}\right\}_{\beta \in B}$ is bounded in $F_{3 / 4}$. (Note that $m_{\alpha} \rightarrow m \Rightarrow\left\|m_{\beta}^{(1)}\right\| \rightarrow\|m\|$.) Furthermore $\left|f_{m_{\alpha}}^{\prime}(k)\right| \leqq 1$, so that $\left\{f_{m_{\alpha}}\right\}$, and hence also $\left\{\left(1+k^{2}\right)^{-3 / 4} f_{m_{\beta}^{(1)}}(k)\right\}_{\beta \in B}$ is equicontinuous. The set $\left\{f_{m_{\beta}^{(1)}}\right\}_{\beta \in B}$ is therefore relatively compact in $F_{3 / 4}$. We conclude that there 
exists a subnet $\left(f_{m_{\kappa}^{(2)}}\right)_{\kappa \in K}$ converging in $F_{3 / 4}$. Let $f \in F_{3 / 4}$ be the limit. We shall prove that $f=f_{m}$. To this end we use

\section{Lemma 6.2.}

$$
\left|\theta_{c}(x-y)-\theta_{c}\left(x^{\prime}-y^{\prime}\right)\right| \leqq\left|\theta_{c}\left(x-x^{\prime}\right)\right|+\left|\theta_{c}\left(y-y^{\prime}\right)\right| .
$$

Proof. If $u, v \geqq 0$ then $\theta_{c}(u+v) \leqq \theta_{c}(u)+\theta_{c}(v)$, because $\theta_{c}(k)$ is concave for $k \geqq 0$. It follows that, for general $u, v \in \mathbf{R},\left|\theta_{c}(u)\right| \leqq\left|\theta_{c}(u-v)\right|+\left|\theta_{c}(v)\right|$ and $\left|\theta_{c}(u)-\theta_{c}(v)\right| \leqq$ $\left|\theta_{c}(u-v)\right| \leqq\left|\theta_{c}(u-v+z)\right|+\left|\theta_{c}(z)\right|$. Now put $u=x-y, v=x^{\prime}-y^{\prime}$, and $z=y-y^{\prime}$.

This lemma implies that $\theta_{c}\left(f_{m_{\kappa}^{(2)}}(k)-f_{m_{\kappa}^{(2)}}(\cdot)\right)$ converges to $\theta_{c}(f(k)-f(\cdot))$ in $F_{3 / 4}$ for all $k \in \mathbf{R}$. Next we use

Lemma 6.3. If $m_{\alpha} \rightarrow m$ in $E$ and $f_{\alpha} \rightarrow f$ in $F_{3 / 4}$, then $\left\langle f_{\alpha}, m_{\alpha}\right\rangle \rightarrow\langle f, m\rangle$.

Proof. $\left|\left\langle f_{\alpha}, m_{\alpha}\right\rangle-\langle f, m\rangle\right| \leqq\left|\left\langle f_{\alpha}-f, m_{\alpha}\right\rangle\right|+\left|\left\langle f, m_{\alpha}\right\rangle-\langle f, m\rangle\right|$. The second term converges to zero because $f \in F$. As to the first term we have: given $\varepsilon>0$, there exists $\alpha_{0}$ such that

But then

$$
\alpha \geqq \alpha_{0} \Rightarrow\left|f_{\alpha}(k)-f(k)\right| \leqq\left(1+k^{2}\right)^{3 / 4} \varepsilon \text { for all } k \in \mathbf{R} .
$$

$$
\left|\left\langle f_{\alpha}-f, m_{\alpha}\right\rangle\right| \leqq \varepsilon \int\left(1+k^{2}\right)^{3 / 4} m_{\alpha}(d k),
$$

and the latter integral is bounded because $m_{\alpha} \rightarrow m$ in $E$ and $\left(1+k^{2}\right)^{3 / 4} \in F$.

We conclude that

$$
k-\int \theta_{c}\left(f_{m_{\kappa}^{(2)}}(k)-f_{m_{\kappa}^{(2)}}\left(k^{\prime}\right)\right) m_{\kappa}^{(2)}\left(d k^{\prime}\right)
$$

converges to

$$
k-\int \theta_{c}\left(f(k)-f\left(k^{\prime}\right)\right) m\left(d k^{\prime}\right)
$$

for all $k \in \mathbf{R}$. On the other hand $f_{m_{k}^{(2)}}(k) \rightarrow f(k)$, so that $f$ satisfies (5.2) and by uniqueness, $f=f_{m}$. The continuity of $m \mapsto f_{m}$ now follows from Lemma 6.1.

Proposition 6.2. The functional $m \mapsto G[m]=\int\left(k^{2}-f_{m}(k)^{2}\right) m(d k)$ is continuous: $E \rightarrow \mathbf{R}$.

Proof. In view of Lemma 6.3 it is sufficient to prove that $m \mapsto g_{m}(k)=k^{2}-f_{m}(k)^{2}$ is continuous: $E \rightarrow F_{3 / 4}$. because

Let $\left(m_{\alpha}\right)_{\alpha \in A}$ be a net in $E$ converging to $m \in E$. Then $f_{m_{\alpha}} \rightarrow f_{m}$ in $F_{3 / 4}$. But $g_{m_{\alpha}} \in F_{3 / 4}$

$$
\left|g_{m_{\alpha}}(k)\right|=\left|k+f_{m_{\alpha}}(k)\right|\left|k-f_{m_{\alpha}}(k)\right| \leqq \pi\left\|m_{\alpha}\right\|\left(2|k|+\pi\left\|m_{\alpha}\right\|\right),
$$

and $\left\|m_{\alpha}\right\|$ is bounded. We also have

$$
g_{m}(k)=2 k \int \theta_{c}\left(f_{m}(k)-f_{m}\left(k^{\prime}\right)\right) m\left(d k^{\prime}\right)+\left[\int \theta_{c}\left(f_{m}(k)-f_{m}\left(k^{\prime}\right)\right) m\left(d k^{\prime}\right)\right]^{2},
$$

hence

$$
\begin{aligned}
g_{m}^{\prime}(k)= & 2 \int \theta_{c}\left(f_{m}(k)-f_{m}\left(k^{\prime}\right)\right) m\left(d k^{\prime}\right)+2 \int \frac{2 c}{c^{2}+\left(f_{m}(k)-f_{m}\left(k^{\prime}\right)\right)^{2}} f^{\prime}(k) m\left(d k^{\prime}\right) \\
& \cdot\left\{k+\int \theta_{c}\left(f_{m}(k)-f_{m}\left(k^{\prime}\right)\right) m\left(d k^{\prime}\right)\right\},
\end{aligned}
$$


so that

$$
\left|g_{m}^{\prime}(k)\right| \leqq 2 \pi\|m\|+\frac{4 \pi}{c}\|m\|^{2}+2|k| \int \frac{2 c}{c^{2}+\left(f_{m}(k)-f_{m}\left(k^{\prime}\right)\right)^{2}} m\left(d k^{\prime}\right) .
$$

The last term is also bounded because, by (5.17),

$$
\left|f_{m}(k)-f_{m}\left(k^{\prime}\right)\right| \geqq\left(1+2 c^{-1}\|m\|\right)^{-1}\left|k-k^{\prime}\right|=\alpha\left|k-k^{\prime}\right|,
$$

and therefore,

$$
\begin{aligned}
& |k| \int \frac{2 c}{c^{2}+\left(f_{m}(k)-f_{m}\left(k^{\prime}\right)\right)^{2}} m\left(d k^{\prime}\right) \leqq|k| \int \frac{2 c}{c^{2}+\alpha^{2}\left(k-k^{\prime}\right)^{2}} m\left(d k^{\prime}\right) \\
& \quad \leqq \int \frac{2 c\left|k-k^{\prime}\right|}{c^{2}+\alpha^{2}\left(k-k^{\prime}\right)^{2}} m\left(d k^{\prime}\right)+\int \frac{2 c\left|k^{\prime}\right|}{c^{2}+\alpha^{2}\left(k-k^{\prime}\right)^{2}} m\left(d k^{\prime}\right) \\
& \quad \leqq \frac{2}{3} \sqrt{2}\left(1+2 c^{-1}\|m\|\right)\|m\|+\frac{2}{c} \int\left|k^{\prime}\right| m\left(d k^{\prime}\right) .
\end{aligned}
$$

We conclude that $\left\{g_{m_{\alpha}}\right\}$ and hence also $\left\{\left(1+k^{2}\right)^{-3 / 4} g_{m_{\alpha}}(k)\right\}$ is equicontinuous. Each subnet therefore has a convergent subnet. But since $f_{m_{\alpha}} \rightarrow f_{m}$ in $F_{3 / 4} \Rightarrow$ $g_{m_{\alpha}}(k) \rightarrow g_{m}(k)$ for all $k \in \mathbf{R}$, we conclude with Lemma 6.1 that $g_{m_{\alpha}} \rightarrow g_{m}$ in $F_{3 / 4}$.

\section{The Yang-Yang Trace Formula}

Apart from being continuous, the function $G[m]$ also has to satisfy condition (1.36) if we want to apply Varadhan's Theorem. In our concrete situation this condition reads

$$
\lim _{A \rightarrow \infty} \limsup _{l \rightarrow \infty} \frac{1}{\beta V_{l}} \ln \int_{\{m \in E \mid G[m] \geqq A\}} \exp \left\{\beta V_{l} G[m]\right\} \mathbf{K}_{l}^{\mu}[d m]=-\infty .
$$

In proving this condition we shall use several times the following basic lemma without always mentioning it:

Lemma 7.1. Let $\left\{a_{l}\right\}$ and $\left\{b_{l}\right\}$ be sequences of positive real numbers. Then

$$
\limsup _{l \rightarrow \infty} \frac{1}{\beta V_{l}} \ln \left(a_{l}+b_{l}\right) \leqq\left(\limsup _{l \rightarrow \infty} \frac{1}{\beta V_{l}} \ln a_{l}\right) \vee\left(\limsup _{l \rightarrow \infty} \frac{1}{\beta V_{l}} \ln b_{l}\right) .
$$

The first time we use this lemma is in splitting the integral in (7.1) into integrals over the regions $E_{<}=\left\{m \in E \mid\|m\|<\rho_{0}\right\}$ and $E_{\geqq}=\left\{m \in E \mid\|m\| \geqq \rho_{0}\right\}$ respectively, where $\rho_{0}$ is such that, for measures $m$ in the support of $\mathbf{K}_{l}^{\mu}$ such that $\bar{\rho} \geqq \rho_{0}$, the bound (5.31) holds; that is,

$$
\bar{\rho} \geqq \rho_{0} \Rightarrow G[m] \leqq \varepsilon-\frac{c}{\pi^{2}} \frac{\varepsilon}{\bar{\rho}} .
$$

The integral over $E_{<}$can be easily bounded with the help of the general bound (5.22). With $\alpha_{0}=\left(1+2 c^{-1} \rho_{0}\right)^{-1}$ we have

$$
\bar{\rho}<\rho_{0} \Rightarrow G[m] \leqq\left(1-\alpha_{0}^{2}\right) \varepsilon .
$$


We use the fact that, for $\delta>0$,

$$
1_{\{m \in E \mid G[m] \geqq A\}} \leqq \exp \left\{\delta \beta V_{l}(G[m]-A)\right\}
$$

to bound the integral over $E_{<}$as follows,

$$
\begin{aligned}
& \frac{1}{\beta V_{l}} \ln \int_{\left\{m \in E_{<} \mid G[m] \geqq A\right\}} \exp \left\{\beta V_{l} G[m]\right\} \mathbf{K}_{l}^{\mu}[d m] \\
& \leqq \frac{1}{\beta V_{l}} \ln \int_{E_{<}} \exp \left\{\beta V_{l}((1+\delta) G[m]-\delta A)\right\} \mathbf{K}_{l}^{\mu}[d m] \\
& \quad=\frac{1}{\beta V_{l}} \ln \int_{E_{<}} \exp \left\{\zeta \beta V_{l} G[m]\right\} \mathbf{K}_{l}^{\mu}[d m]-\delta A,
\end{aligned}
$$

with $\zeta=1+\delta$. Hence, in order that

$$
\limsup _{l \rightarrow \infty} \frac{1}{\beta V_{l}} \ln \int_{\left\{m \in E_{<} \mid G[m] \geqq A\right\}} \exp \left\{\beta V_{l} G[m]\right\} \mathbf{K}_{l}^{\mu}[d m]
$$

approaches $-\infty$ as $A \rightarrow \infty$, it is sufficient that, for some $\zeta>1$,

$$
\limsup _{l \rightarrow \infty} \frac{1}{\beta V_{l}} \ln \int_{E_{<}} \exp \left\{\zeta \beta V_{l} G[m]\right\} \mathbf{K}_{l}^{\mu}[d m]<\infty .
$$

Now let $\zeta=\left(1-\alpha_{0}^{2} / 2\right) /\left(1-\alpha_{0}^{2}\right)$. Then

$$
\begin{aligned}
\frac{1}{\beta V_{l}} \ln \int_{E_{<}} \exp \left\{\zeta \beta V_{l} G[m]\right\} \mathbf{K}_{l}^{\mu}[d m] & \leqq \frac{1}{\beta V_{l}} \ln \int \exp \left\{\zeta \beta V_{l}\left(1-\alpha_{0}^{2}\right) \varepsilon\right\} \tilde{K}_{l}^{\mu}(d \bar{\rho}, d \varepsilon) \\
& =\widetilde{C}_{l}^{\mu}\left(0,1-\frac{1}{2} \alpha_{0}^{2}\right) \rightarrow\left(\frac{1}{2} \alpha_{0}^{2}\right)^{-1 / 2} p^{0}(\mu)-p^{0}(\mu) .
\end{aligned}
$$

The integral over $E_{\geqq}$is more difficult to control. Indeed, the simple-minded approach in the proof of Proposition 5.3 does not work because, if we put $N=\lambda L$ with $L$ large enough so that the bound (5.31) holds, the corresponding term in the bound (5.36) becomes of the order $L^{\lambda L} e^{\text {const. } L}$. We improve on this by iterative use of the following inequality,

Lemma 7.2. Let $\sigma>0$ be fixed. Then, for arbitrary integer $k>0$ and arbitrary real $n \geqq 0$,

$$
\begin{aligned}
& \sum_{m=0}^{\infty} \exp \left\{-\sigma\left[(n+m)^{2}+\cdots+(n+m+k)^{2}\right]\right\} \\
& \quad \leqq\left\{1+\frac{1}{\sigma(k+1)(2 n+k)}\right\} \exp \left\{-\sigma\left[n^{2}+(n+1)^{2}+\cdots+(n+k)^{2}\right]\right\} .
\end{aligned}
$$

Proof. We have

$$
\begin{aligned}
\sum_{m=0}^{\infty} \exp \left\{-\sigma\left[(n+m)^{2}+\cdots+(n+m+k)^{2}\right]\right\} \\
=\exp \left[-\sigma \sum_{i=0}^{k}(n+i)^{2}\right] . \\
\quad \cdot \sum_{m=0}^{\infty} \exp \left\{-\sigma(k+1)\left[m^{2}+(2 n+k) m\right]\right\}
\end{aligned}
$$




$$
\begin{aligned}
= & \exp \left[-\sigma \sum_{i=0}^{k}(n+i)^{2}+\sigma(k+1)\left(n+\frac{1}{2} k\right)^{2}\right] . \\
& \cdot \sum_{m=0}^{\infty} \exp \left\{-\sigma(k+1)\left(m+n+\frac{1}{2} k\right)^{2}\right\} \\
\leqq & \exp \left[-\sigma \sum_{i=0}^{k}(n+i)^{2}\right] . \\
& \cdot\left\{1+e^{\sigma(k+1)(n+k / 2)^{2}} \int_{n+k / 2}^{\infty} e^{-\sigma(k+1) x^{2}} d x\right\} \\
\leqq & \exp \left\{-\sigma\left[n^{2}+\cdots+(n+k)^{2}\right]\right\}\left\{1+\frac{1}{\sigma(k+1)(2 n+k)}\right\} .
\end{aligned}
$$

Iterating this inequality we obtain

Lemma 7.3. Let $n \geqq 0, N \geqq 1$. Then

$$
\begin{aligned}
& \sum_{n_{1} \geqq n} \sum_{n_{2} \geqq n_{1}+1} \cdots \sum_{n_{N} \geqq n_{N-1}+1} \exp \left[-\sigma \sum_{i=1}^{N} n_{i}^{2}\right] \\
& \leqq \prod_{k=1}^{N}\left\{1+\frac{1}{\sigma k(2 n-1+2 N-k)}\right\} \cdot \exp \left[-\sigma \sum_{i=0}^{N-1}(n+i)^{2}\right] .
\end{aligned}
$$

Proof. We use induction on $N$. If $N=1$ then

$$
\sum_{n_{1} \geqq n} \exp \left[-\sigma n_{1}^{2}\right] \leqq\left\{1+\frac{1}{2 \sigma n}\right\} \exp \left[-\sigma n^{2}\right]
$$

(which, of course, is only useful if $n>0$ ). Now suppose that statement is true for a given $N$. Then

$$
\begin{aligned}
\sum_{n_{1} \geqq n} & \sum_{n_{2} \geqq n_{1}+1} \cdots \sum_{n_{N}+1 \geqq n_{N}+1} \exp \left[-\sigma \sum_{i=1}^{N+1} n_{i}^{2}\right] \\
\leqq & \sum_{n_{1} \geqq n} \exp \left[-\sigma n_{1}^{2}\right] \prod_{k=1}^{N}\left\{1+\frac{1}{\sigma k\left(2 n_{1}+1+2 N-k\right)}\right\} . \\
& \cdot \exp \left\{-\sigma\left[\left(n_{1}+1\right)^{2}+\cdots+\left(n_{1}+N\right)^{2}\right]\right\} \\
\leqq & \prod_{k=1}^{N}\left\{1+\frac{1}{\sigma k(2 n+1+2 N-k)}\right\} . \\
& \cdot\left\{1+\frac{1}{\sigma(N+1)(2 n+N)}\right\} \exp \left\{-\sigma\left[n^{2}+\cdots+(n+N)^{2}\right]\right\} \\
= & \prod_{k=1}^{N+1}\left\{1+\frac{1}{\sigma k(2 n+1+2 N-k)}\right\} \cdot \exp \left[-\sigma \sum_{i=0}^{N}(n+i)^{2}\right] .
\end{aligned}
$$


Proposition 7.1. The function $G[m]=\int\left(k^{2}-f_{m}(k)^{2}\right) m(d k)$ satisfies the conditions for Varadhan's Theorem, in particular (7.1).

Proof. We have shown in Sect. 6 that $G[m]$ is continuous. Given the above bounds on the region $E_{<}$of the integral (7.1), it remains to show that

$$
\lim _{A \rightarrow \infty} \limsup _{l \rightarrow \infty} \frac{1}{\beta V_{l}} \int_{\left\{m \in E_{\geqq} \mid G[m] \geqq A\right\}} \exp \left\{\beta V_{l} G[m]\right\} \mathbf{K}_{l}^{\mu}[d m]=-\infty .
$$

Using (7.2) we have

$$
\begin{aligned}
& \frac{1}{\beta V_{l}} \ln \int_{\left\{m \in E_{\geqq} \mid G[m] \geqq A\right\}} \exp \left\{\beta V_{l} G[m]\right\} \mathbf{K}_{l}^{\mu}[d m] \\
& \quad \leqq \frac{1}{\beta V_{l}} \ln \int_{\left\{(\bar{\rho}, \varepsilon) \mid \varepsilon \geqq A ; \bar{\rho} \geqq \rho_{0}\right\}} \exp \left\{\beta V_{l}\left(\varepsilon-\frac{c}{\pi^{2}} \frac{\varepsilon}{\bar{\rho}}\right)\right\} \tilde{\mathbf{K}}_{l}^{\mu}(d \bar{\rho}, d \varepsilon) \\
& \quad=\frac{1}{\beta V_{l}} \ln \sum_{\left\{\sigma \in \Omega \mid \varepsilon_{l}(\sigma) \geqq A ; \bar{\rho}_{l}(\sigma) \geqq \rho_{0}\right\}} \exp \left\{\beta \mu V_{l} \bar{\rho}_{l}(\sigma)-\frac{c}{\pi^{2}} \beta V_{l} \frac{\varepsilon_{l}(\sigma)}{\bar{\rho}_{l}(\sigma)}\right\}-p_{l}^{0}(\mu),
\end{aligned}
$$

where $\bar{\rho}_{l}(\sigma)=\left(1 / V_{l}\right) \sum_{n \in \mathbf{Z}} \sigma_{n}$, and $\varepsilon_{l}(\sigma)=\left(1 / V_{l}\right) \sum_{n \in \mathbf{Z}} k_{n}(\sigma)^{2} \sigma_{n}$.

Clearly,

$$
\varepsilon_{l}(\sigma) \geqq \frac{1}{V_{l}}\left(\frac{2 \pi}{V_{l}}\right)^{2} \sum_{n=-(N-1) / 2}^{(N-1) / 2} n^{2}=\frac{1}{3} \pi^{2} \bar{\rho}_{l}^{3}(\sigma)-\frac{1}{3} \frac{\pi^{2}}{N^{2}} \bar{\rho}_{l}^{3}(\sigma) \geqq \frac{1}{4} \pi^{2} \bar{\rho}_{l}^{3}(\sigma) \quad(N \geqq 2) .
$$

Therefore,

$$
\frac{\varepsilon_{l}(\sigma)}{\bar{\rho}_{l}(\sigma)} \geqq\left(\frac{\pi^{2}}{4}\right)^{1 / 3} A^{2 / 3} \equiv D,
$$

and hence

$$
\begin{aligned}
& \sum_{\left\{\sigma \in \Omega \mid \varepsilon_{l}(\sigma) \geqq A ; \bar{\rho}_{l}(\sigma) \geqq \rho_{0}\right\}} \exp \left\{\beta \mu \sum_{n \in \mathbf{Z}} \sigma_{n}-\frac{c}{\pi^{2}} \beta V_{l} \frac{\varepsilon_{l}(\sigma)}{\bar{\rho}_{l}(\sigma)}\right\} \\
& \leqq \sum_{\left\{\sigma \in \Omega: \sum_{n \in \mathbf{Z}} \sigma_{n} \geqq 2\right\}} \exp \left\{\beta \mu \sum_{n \in \mathbf{Z}} \sigma_{n}-\frac{c}{\pi^{2}} \beta V_{l}\left((1-\delta) \frac{\varepsilon_{l}(\sigma)}{\bar{\rho}_{l}(\sigma)}+\delta D\right)\right\},
\end{aligned}
$$

using the same upper bound as in (7.4) for the indicator function. It follows that it suffices to show that

$$
\frac{1}{\beta V_{l}} \ln \sum_{\left\{\sigma \in \Omega: \sum_{n \in \mathbf{Z}} \sigma_{n} \geqq 2\right\}} \exp \left\{\beta \mu \sum_{n \in \mathbf{Z}} \sigma_{n}-\frac{c}{\pi^{2}}(1-\delta) \beta V_{l} \frac{\varepsilon_{l}(\sigma)}{\bar{\rho}_{l}(\sigma)}\right\}
$$

is bounded as $l \rightarrow \infty$. (Comp. (7.5)) Writing $L=V_{l}$ and $\kappa=4 c(1-\delta) \beta$ this expression becomes

$$
\frac{1}{\beta L} \ln \sum_{N=2}^{\infty} e^{\beta \mu N} \sum_{\left\{n_{j}\right\}_{j=1}^{N}} \exp \left\{-\frac{\kappa}{L N} \sum_{j=1}^{N} n_{j}^{2}\right\}
$$


where the sum is over all sets of $N$ distinct integers or half-odd integers according as $N$ is odd or even. In this sum we replace the negative $n_{j}$ 's by their absolute value. Each $n_{j}>0$ can then occur once or twice. We distinguish these cases by additional variables $\eta_{j}$ which can take the values 1 or 2 . Thus we obtain

$$
\begin{aligned}
& \sum_{N=2}^{\infty} e^{\beta \mu N} \sum_{\left\{n_{j}\right\}_{j}^{N}=1} \exp \left\{-\frac{\kappa}{L N} \sum_{j=1}^{N} n_{j}^{2}\right\} \\
& \leqq \sum_{N=2}^{\infty} \sum_{\left\{\eta_{j}\right\}_{j=1}^{N}} \exp \left\{\beta \mu \sum_{j=1}^{N} \eta_{j}\right\} \sum_{\left\{n_{j} \geqq 0\right\}_{j=1}^{N}} \exp \left\{-\frac{\kappa}{L \sum \eta_{j}} \sum_{j=1}^{N} \eta_{j} n_{j}^{2}\right\} \\
& \leqq \sum_{N=2}^{\infty} 2^{N} e^{2 \beta \mu N} \sum_{\left\{n_{j} \geqq 0\right\}_{j=1}^{N}} \exp \left\{-\frac{\kappa}{2 L N} \sum_{j=1}^{N} n_{j}^{2}\right\} .
\end{aligned}
$$

Next we use Lemma 7.3 to estimate the last sum in this expression:

$$
\begin{aligned}
\sum_{\left\{n_{j} \geqq 0\right\}_{j=1}^{N}} \exp \left\{-\frac{\kappa}{2 L N} \sum_{j=1}^{N} n_{j}^{2}\right\} & =\sum_{n_{1} \geqq 0} \sum_{n_{2} \geqq n_{1}+1} \ldots \sum_{n_{N} \geqq n_{N-1}+1} \exp \left\{-\frac{\kappa}{2 L N} \sum_{j=1}^{N} n_{j}^{2}\right\} \\
& \leqq \prod_{k=1}^{N}\left\{1+\frac{2 L N}{\kappa k(2 N-k-1)}\right\} \exp \left\{-\frac{\kappa}{2 L N} \sum_{n=1}^{N-1} n^{2}\right\} \\
& \leqq(1+2 \tilde{L}) \prod_{k=1}^{N-1}\left(1+\frac{\tilde{L}}{k}\right) \exp \left\{-\frac{1}{3 \tilde{L} N}(N-1)^{3}\right\}
\end{aligned}
$$

where $\tilde{L}=2 L / \kappa$. We assume $\tilde{L} \geqq 1$. It remains to estimate

$$
3 \tilde{L} \sum_{N=2}^{\infty}\left(2 e^{2 \beta \mu}\right)^{N} \prod_{k=1}^{N-1}\left(1+\frac{\tilde{L}}{k}\right) \exp \left\{-\frac{1}{3 \tilde{L} N}(N-1)^{3}\right\} .
$$

Again we split this sum into two parts (using Lemma 7.1) and distinguish the cases $N-1 \leqq[\tilde{L}]$ and $N \geqq[\tilde{L}]+2$. In the first case we write

$$
\prod_{k=1}^{N-1}\left(1+\frac{\tilde{L}}{k}\right) \leqq 2^{N-1} \frac{\tilde{L}^{N-1}}{(N-1) !} \leqq(2 e)^{N-1}\left(\frac{\tilde{L}}{N-1}\right)^{N-1} \leqq(2 e)^{N-1} e^{\tilde{L} / e}
$$

Inserting we find

$$
\begin{aligned}
& \frac{1}{\beta L} \ln \left\{3 \tilde{L} \sum_{N=2}^{[\tilde{L}]+1}\left(2 e^{2 \beta \mu}\right)^{N} \prod_{k=1}^{N-1}\left(1+\frac{\tilde{L}}{k}\right) \exp \left[-\frac{1}{3 \tilde{L} N}(N-1)^{3}\right]\right\} \\
& \leqq \frac{1}{\beta L} \ln \left\{3 \tilde{L}(2 e)^{[\tilde{L}]} e^{\tilde{L} / e}[\tilde{L}]\left(2 e^{2 \beta \mu}\right)^{[\tilde{L}]+1}\right\},
\end{aligned}
$$

which tends to

$$
\frac{2}{\beta \kappa}\left(1+2 \ln 2+\frac{1}{e}+2 \beta \mu\right)<\infty .
$$

In the second case we write 


$$
\begin{aligned}
\prod_{k=1}^{N-1}\left(1+\frac{\tilde{L}}{k}\right) & =\prod_{k=1}^{[\tilde{L}]}\left(1+\frac{\tilde{L}}{k}\right) \prod_{k=[\tilde{L}]+1}^{N-1}\left(1+\frac{\tilde{L}}{k}\right) \leqq 2^{[\tilde{L}]} \frac{\tilde{L}}{[\tilde{L}] !} \exp \left\{\tilde{L}_{k=[\tilde{L}]+1}^{N-1} \frac{1}{k}\right\} \\
& \leqq(2 e)^{[\tilde{L}]} \exp \left\{\tilde{L} \ln \frac{N-1}{[\tilde{L}]}\right\}=(2 e)^{[\tilde{L}]}\left(\frac{N-1}{[\tilde{L}]}\right)^{L} \leqq(4 e)^{\tilde{L}} e^{(N-1) / e}
\end{aligned}
$$

This yields

$$
\begin{aligned}
& \frac{1}{\beta L} \ln \left\{3 \tilde{L} \sum_{N=\tilde{[}]+2}^{\infty}\left(2 e^{2 \beta \mu}\right)^{N} \prod_{k=1}^{N-1}\left(1+\frac{\tilde{L}}{k}\right) \exp \left[-\frac{1}{3 \tilde{L} N}(N-1)^{3}\right]\right\} \\
& \quad \leqq \frac{1}{\beta L} \ln \left\{3 \tilde{L}(4 e)^{\tilde{L}} \sum_{N=[\tilde{L}]+2}^{\infty}\left(2 e^{2 \beta \mu}\right)^{N} e^{N / e} \exp \left(-\frac{1}{3 \tilde{L}} N^{2}+\frac{1}{\widetilde{L}} N\right)\right\} \\
& \leqq \frac{1}{\beta L} \ln \left\{3 \tilde{L}(4 e)^{\tilde{L}} \sum_{N=[\tilde{L}]+2}^{\infty} \exp \left(\lambda N-\frac{1}{3 \tilde{L}} N^{2}\right)\right\}
\end{aligned}
$$

where

$$
\lambda=2 \beta \mu+\ln 2+\frac{1}{e}+1 \geqq 2 \beta \mu+\ln 2+\frac{1}{e}+\frac{1}{\tilde{L}} .
$$

Now

$$
\sum_{N=[\tilde{L}]+2}^{\infty} \exp \left\{\lambda N-\frac{1}{3 \widetilde{L}} N^{2}\right\} \leqq \exp \left(\frac{3}{4} \lambda^{2}[\tilde{L}]\right) \sum_{N=-\infty}^{\infty} e^{-N^{2} / \tilde{L}} \leqq(1+\sqrt{3 \pi \widetilde{L}}) \exp \left\{\frac{3}{4} \lambda^{2} \tilde{L}\right\}
$$

We conclude that the lim sup of the left-hand side of (7.16) is bounded by

$$
\frac{2}{\beta \kappa}\left(4 e+\frac{3}{4} \lambda^{2}\right)<\infty \text {. }
$$

Using Proposition 7.1, Theorem 4.2 and Varadhan's Theorem, we find from (1.34) that

$$
p(\mu)=\lim _{l \rightarrow \infty} p_{l}(\mu)=p^{0}(\mu)+\sup _{m \in E}\left\{G[m]-I^{\mu}[m]\right\} .
$$

This reduces to

$$
p(\mu)=\sup _{m \in E}\{\mu\|m\|-f[m]\}
$$

using (1.33), (1.37) and (1.38); the formula

$$
p(\mu)=\beta^{-1} \int_{\mathbf{R}} \ln \left(1+e^{-\beta \varepsilon(k ; \beta, \mu)}\right) \frac{d k}{2 \pi},
$$

where $\varepsilon(; \beta, \mu)$ is given by (1.43) then follows using standard methods of the calculus of variations as in [1], and the proof of the theorem stated at the end of Sect. 1 is complete.

\section{References}

1. Yang, C. N., Yang, C. P.: Thermodynamics of a one-dimensional system of Bosons with repulsive delta-function interaction. J. Math. Phys. 10, 1115-1122 (1969) 
2. Lieb, E. H., Liniger, W.: Exact analysis of an interacting bose gas. I: General solution and the ground state, II: The exitation spectrum. Phys. Rev. 130, 1605-1624 (1963)

3. Lowenstein, J. H.: Introduction to the Bethe Ansatz approach to $(1+1)$-dimensional models. In: Recent advances in field theory and statistical mechanics. Les Houches Summer School 1982. Zuber, J.-B., Stora, R. (eds.). Amsterdam: North-Holland 1984

4. Thacker, H. B.: Exact integrability in quantum field theory and statistical systems. Rev. Mod. Phys. 53, 253-285 (1981)

5. Andrei, N.: Diagonalization of the Kondo Hamiltonian. Phys. Rev. Lett. 45, 379-382 (1980)

6. Wiegmann, P. B.: Exact solution of $s-d$ exchange model at $T=0$. J.E.T.P. Lett. 31, 364-370 (1980)

7. Andrei, N., Furuya, K., Lowenstein, J. H.: Solution of the Kondo problem. Rev. Mod. Phys. 55, 331-402 (1983)

8. De Vega, H. J., Karowski, M.: Conformal invariance and integrable theories. Nucl. Phys. B285 [FS 19], 619-638 (1987)

9. Landau, L. D., Lifschitz, E. M.: Statistical physics; Volume 5 of Course of Theoretical Physics. Transl. Peierls, E., Peierls, R. F. (eds.). London-Paris: Pergamon Press 1958

10. Varadhan, S. R. S.: Asymptotic probabilities and differential equations. Commun. Pure Appl. Math. 19, 261-286 (1966)

11. Lebowitz, J. L., Rose, H. A., Speer, E. R.: Statistical mechanics of the non-linear Schrödinger Equation. J. Stat. Phys. 50, 657-687 (1988)

12. Berg, M. van den, Lewis, J. T., Pulé, J. V.: A general theory of Bose-Einstein condensation. Helv. Phys. Acta 59, 1271-1288 (1986)

13. Lewis, J. T., Pulé, J. V., Zagrebnov, V. A.: The large deviation principle for the Kac distribution. Helv. Phys. Acta 61, 1063-1078 (1988)

14. Barbu, V., Precupanu, Th.: Convexity and optimization in Banach spaces Alphen aanden Rijn: Sijthoff \& Noordhoff 1975

15. Ellis, R. S.: Entropy, large deviations and statistical mechanics. Berlin, Heidelberg, New York: Springer 1985

16. Berg, M. van den, Lewis, J. T., Pulé, J. V.: The large deviation principle and some models of an interacting bose gas. Commun. Math. Phys. 118, 61-85 (1988)

Communicated by J. Fröhlich

Received November 28, 1988 